\title{
Impulse-Regime Analysis of Novel Optically-Inspired Phenomena at Microwaves
}

\author{
J. Sebastian Gomez-Diaz ${ }^{1}$, Alejandro Alvarez-Melcon ${ }^{1}$, \\ Shulabh Gupta ${ }^{2}$ and Christophe Caloz ${ }^{2}$ \\ ${ }^{1}$ Universidad Politécnica de Cartagena \\ ${ }^{2}$ École Polytechnique de Montréal \\ ${ }^{1}$ Spain \\ ${ }^{2}$ Canada
}

\section{Introduction}

The ever increasing of needs for high data-rate wireless system is currently producing a shift from narrow-band radio towards ultra-wideband (UWB) radio operation [see Ghavami et al. (2007)]. Novel microwave tools, concepts, phenomena and direct applications must be developed to meet this demand. While the past decades have been focused on the "magnitude engineering" and filter design [see Pozar (2005)], there is a renewed interest in the "dispersion engineering". In the dispersion engineering approach, the phase is engineered to met various specifications within a given frequency range, so as to process signals in real time.

In this context, the development of electromagnetic metamaterials over the last years [see Caloz \& Itoh (2006) or Marques et al. (2008)], with their intrinsically dispersive nature and subsequent impulse-regime properties, may provide novel and original solutions (see Fig. 1). Metamaterials can easily be synthesized in planar technology under the form of composite right/left-handed (CRLH) transmission lines (TLs), using non-resonant [see Caloz \& Itoh (2006)] or resonant [see Duran-Sindreu et al. (2009)] approaches. These structures have provided novel and exciting applications, such as multi-band components, diplexers, couplers, phase-shifters, power-dividers or antennas with enhanced features, to mention just a few [see Caloz (2009) or Eleftheriades (2009) for a recent review]. However, CRLH structures have mostly been analyzed in the harmonic regime to date, and therefore only a few impulse-regime components and systems have been proposed so far. An example of these applications is the tunable pulse delay line presented in in Abielmona et al. (2007).

In this chapter, we present recent advances based on Fourier transformation techniques to model dispersive UWB phenomena and far-field radiation from complex CRLH structures. Section 2 first employs inverse Fourier transforms to study pulse propagation along this type of medium. Then, a Fourier transform approach is applied to the current which flows along the CRLH line, accurately retrieving the time-domain far-field radiation of the structure [which behaves as a leaky-wave antenna, (LWA)]. The main advantages of the proposed techniques are the easy treatment of complex CRLH structures, a deep insight into the physics of the phenomena, and an accurate and a fast computation, which avoids the time-consuming analysis required by completely numerical simulations. 


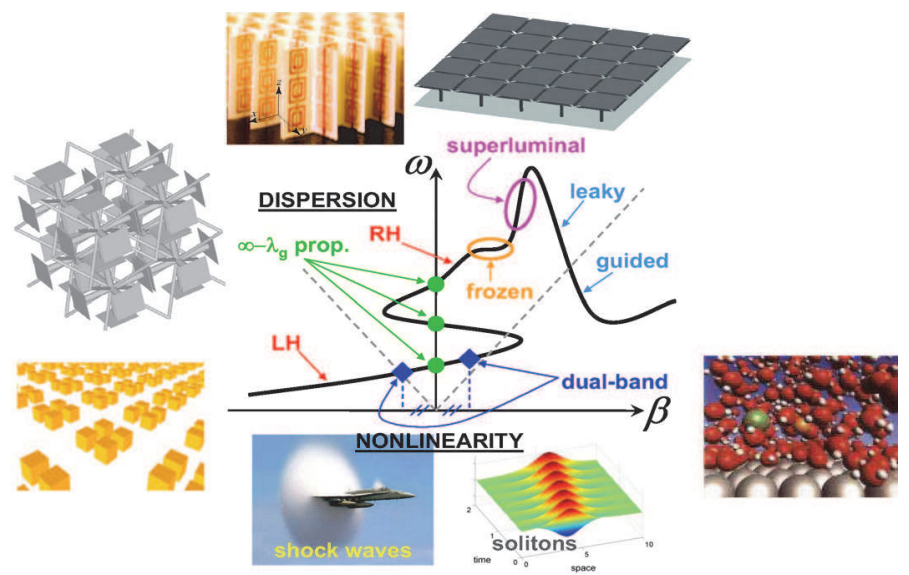

Fig. 1. Illustration of the dispersion engineering concept using metamaterial and CRLH structures. Reprinted with permission from Abielmona et al. (2008). Copyright 2008, URSI.

Section 3 applies the previously derived theory to study the impulse-regime phenomenology of CRLH structures, and subsequently demonstrates several optically-inspired phenomena and applications at microwaves in both the guided and the radiative regime. This study is divided into two main groups, according to the guided-wave or radiative-wave natures of the proposed phenomena and applications.

In the guided-regime, the dispersive properties of pulse propagation along a CRLH line and the temporal Talbot effect, which allows the period rate multiplication of an input pulse train [see Gómez-Díaz et al. (2009b)], will be described and analyzed.

In the radiative regime, the spectral-spatial decomposition property of CRLH LWAs will be exploited to characterize both, in time and in frequency, unknown fast-transient input signals. For this purpose, two different systems (namely, a real time spectrogram analyzer (RTSA) [see Gupta, Abielmona \& Caloz (2009)], and a frequency resolved electrical gating (FREG) [see Gupta, Gómez-Díaz \& Caloz (2009)]) will be carefully studied and simulated.

In all cases, the proposed theory and phenomena will be validated by using simulation results from full-wave commercial softwares and measurements, from fabricated prototypes. Therefore, the usefulness of the Fourier transform approach will be fully demonstrated as an essential mathematical tool for the fast and accurate modeling of very complex UWB structures and phenomena.

\section{Impulse-regime analysis of CRLH structures using a Fourier transform approach}

Propagation of electromagnetic short pulses in complex media, see Felsen (1969), has been a field of great interest for a long time. Most practical developments for pulse propagation in dispersive media have been carried out for optical systems, including optical fibers, couplers, switches and soliton devices [see Saleh \& Teich (2007)]. At microwaves, pulse propagation has been far less studied, and the temporal analysis of dispersive metamaterial structures is usually performed with time-domain full-wave methods. However, these accurate techniques require a high computational cost, due to the meshing of the whole geometry under study. 
In this section, a general time-domain Green's function approach is presented for the analysis of pulse propagation in electrically thin CRLH TLs. This method is based on the transient analysis of 1D transmission lines [see Paul (2007)] combined for the first time with CRLH TL concepts introduced in Caloz \& Itoh (2006). With this equivalent transmission line simplification of the geometry, the Green's functions are available in closed-form, and directly correspond to the voltages and currents along the transmission line. The main advantages of this approach are the unconditional stability and fast computation, due to the continuous treatment of time, and the insight into the physical phenomena provided by the Green's functions. Subsequently, the method is extended to analyze impulse-regime CRLH leaky-wave antennas. The approach is based on the use of the time-domain current which flows along the structure to compute the far-field radiation of the antenna. This technique is especially appropriate to characterize complex radiated-wave UWB phenomena and devices, as it will be demonstrated in Section 3.

\subsection{Composite right/left-handed structures}

The introduction of metamaterials [see Caloz \& Itoh (2006) or Marques et al. (2008)] in the last decade has paved the road to the development of new devices and applications based on the novel fundamental features and phenomena associated to this type of media. Among the most useful metamaterials, one can find the CRLH transmission lines [see Caloz \& Itoh (2006)]. This type of transmission lines, which are inherently nonresonant and low-loss, can be easily implemented in planar technology (such as microstrip or coplanar waveguide, for instance) and provides a practical realization of electromagnetic metamaterials. As any metamaterial, CRLH TLs are generally periodic structures formed by the repetition of unit-cells (an example of this type of cells is shown in Fig. 2) whose size, $p$, must fulfill the condition $p \ll \lambda_{g}$ (where $\lambda_{g}$ is the guided wavelength) ir order to emulate an effectively homogeneous material. A powerful method to analyze these metamaterial lines is the TL approach, presented in Caloz \& Itoh (2006), which employs an ABCD-matrix technique of periodically arranged unit-cells to model the artificial transmission line (see Fig. 3) and to determine its wave propagation characteristics (such as propagation constant or Bloch impedances, see Fig. 4).

There are many examples of interesting and groundbreaking applications of planar TL metamaterials at microwaves, such as for instance multi-band components, filters and diplexers, couplers, power-dividers, phase-shifters, lenses, or backfire to endfire leaky-wave antennas. A review of these and much more applications and devices can be found in metamaterials textbooks, such as in Eleftheriades \& Balmain (2005), in Caloz \& Itoh (2006), or in Marques et al. (2008). Note that all previously mentioned and most of metamaterials applications operate in the harmonic regime up to now, and they have been designed for narrow-band components and systems (even though some of them may support a multi-band operation).

\subsection{Impulse regime analysis of CRLH transmission lines}

Many media, ranging from traditional purely right-handed materials to recent CRLH metamaterials [see Caloz \& Itoh (2006)], can be advantageously analyzed by the transmission line theory described in Pozar (2005). So far, this theory has been applied mostly in the harmonic regime, where Green's functions for both the voltage and the current along the line are available. However, it may also be employed in the time-domain, where the Green's function approach provides an efficient tool to analyze impulse regime signals along 


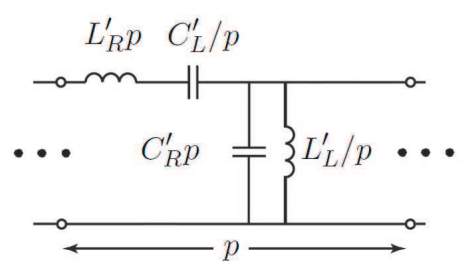

(a)

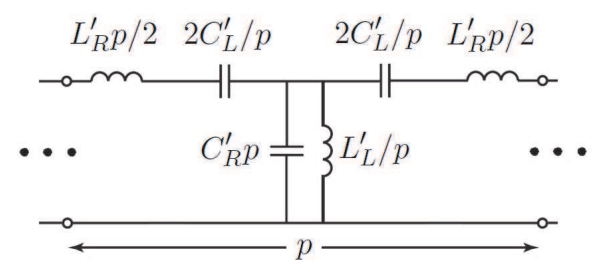

(b)

Fig. 2. Equivalent unit cell circuit model of a lossless CRLH transmission line. (a) Asymmetric configuration. (b) Symmetric configuration.

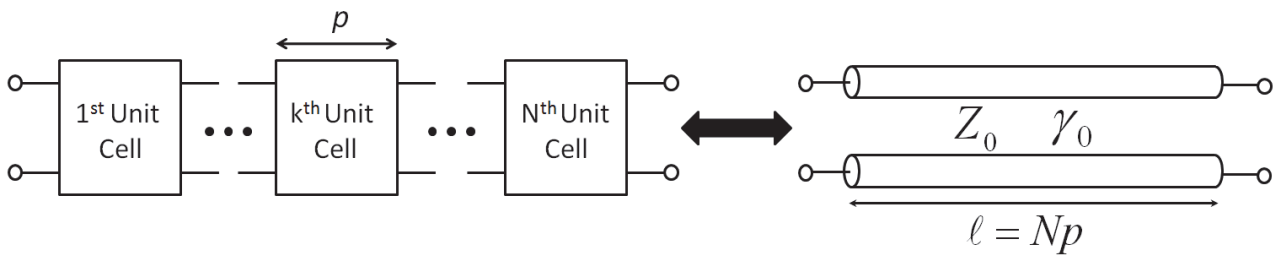

Fig. 3. Equivalence between $N$ cascaded unit cells and a transmission line of length $\ell$, characterized by an equivalent complex propagation constant $\gamma_{0}$ and Bloch impedance $Z_{0}$.

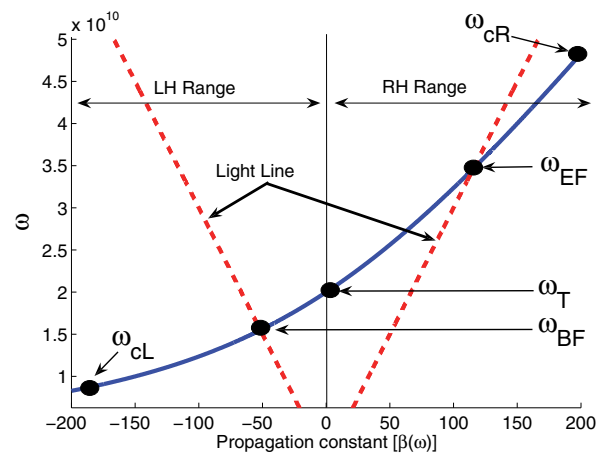

(a)

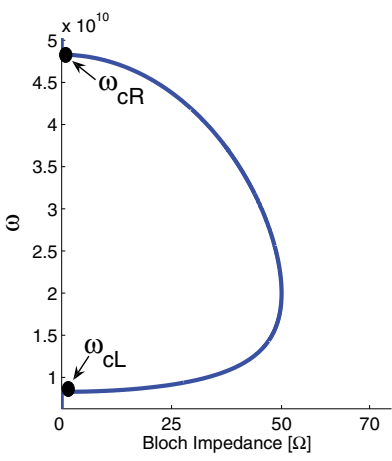

(b)

Fig. 4. Dispersion diagram (a) and frequency-dependent Bloch impedance (b) related to a balanced CRLH unit cell. The size of the unit cell is $p=1 \mathrm{~cm}$ and its circuital parameters are $C_{R}=C_{L}=1.0 \mathrm{pF}$ and $L_{L}=L_{R}=2.5 \mathrm{nH}$. The frequencies $\omega_{c L}$ and $\omega_{c R}$ denote the bandpass frequency region of the line.

transmission lines. In this case, the point source model accurately characterizes a pulse generator, and the computed quantities are the voltages and currents along the line as a function of time.

Consider an electric source $\vec{J}(\vec{r}, t)$ placed in an arbitrary homogeneous and dispersive medium. The wave equation in this case reads [see Collin (1991)]

$$
\nabla \times \nabla \times \vec{E}(\vec{r}, t)+\mu \varepsilon \frac{\partial^{2}}{\partial t^{2}} \vec{E}(\vec{r}, t)=-\mu \frac{\partial}{\partial t} \vec{J}(\vec{r}, t) .
$$


The spatial-temporal dyadic Green's function $\overline{\bar{G}}\left(\vec{r}, \vec{r}^{\prime} ; t, t^{\prime}\right)$ in this equation for a specific medium and a specific source is obtained as the response to a unitary point source $\vec{J}\left(\vec{r}^{\prime}, t^{\prime}\right)=$ $\delta\left(\vec{r}^{\prime} ; t^{\prime}\right)$. As described in Barton (1989), once this Green's function is known, the electric field may be computed using

$$
\vec{E}(\vec{r}, t)=\iint \overline{\bar{G}}\left(\vec{r},{\overrightarrow{r_{g}}}^{\prime} ; t, t^{\prime}\right) \cdot \vec{J}\left({\overrightarrow{r_{g}}}^{\prime}, t^{\prime}\right) d{\overrightarrow{r_{g}}}^{\prime} d t^{\prime},
$$

where the spatial-temporal Green's function may be expressed in terms of its inverse Fourier transform

$$
\overline{\bar{G}}\left(\vec{r},{\overrightarrow{r_{g}}}^{\prime} ; t, t^{\prime}\right)=\frac{1}{2 \pi} \int_{-\infty}^{+\infty} \tilde{\bar{G}}\left(\vec{r},{\overrightarrow{r_{g}}}^{\prime} ; \omega\right) e^{j \omega\left(t-t^{\prime}\right)} d \omega
$$

Inserting Eq. (3) into Eq. (2), yields

$$
\vec{E}(\vec{r}, t)=\frac{1}{2 \pi} \iiint \tilde{\bar{G}}\left(\vec{r}, \overrightarrow{r g}^{\prime} ; \omega\right) \cdot \vec{J}\left(\vec{r}_{g}^{\prime}, t^{\prime}\right) e^{j \omega\left(t-t^{\prime}\right)} d r_{g}^{\prime} d t^{\prime} d \omega .
$$

This expression provides the field radiated by an arbitrary source (in space and time) in an arbitrary dispersive homogenous medium. Since the spatial-temporal distribution of the source is generally known, only the Green's function needs to be computed to provide the field solution. An analogous formulation may naturally be obtained for the magnetic field.

In case of electrically thin 1D transmission lines (placed along the $z$ direction, as shown in Fig. 5), the generator source may be reduced to a point source, greatly reducing the complexity of the problem. Consider a point source placed at the position $\vec{r}_{g}$, and with a temporal dependence

$$
\vec{J}\left(\vec{r}_{g}, t^{\prime}\right)=\vec{\kappa}\left(\vec{r}_{g}\right) I_{g}\left(t^{\prime}\right)=\delta\left(\vec{r}-\vec{r}_{g}\right) I_{g}\left(t^{\prime}\right) \hat{e}_{z}
$$

In this case Eq. (4) is reduced to

$$
\vec{E}(\vec{r}, t)=\frac{1}{2 \pi} \iint \tilde{\bar{G}}\left(\vec{r}, \vec{r}_{g}{ }^{\prime} ; \omega\right) \cdot I_{g}\left(t^{\prime}\right) \hat{e}_{z} e^{j \omega\left(t-t^{\prime}\right)} d t^{\prime} d \omega .
$$

At this point, we use the Fourier transform of the temporal source $\left[\tilde{I}_{g}(\omega)=\mathfrak{F}\left\{I_{g}\left(t^{\prime}\right)\right\}\right.$, where the operator $\mathfrak{F}$ denotes a Fourier transform, see Pipes \& Harvill (1971)]. Note that the input pulse is usually modulated at a frequency $\omega_{0}$, which is included in the $\tilde{I}_{g}(\omega)$ notation as a $e^{j \omega_{0} t}$ term. Besides, a transmission-line Green's function is used to obtain the voltage $(V)$ or the current $(I)$ along the 1D line, which may be expressed as

$$
X(z, t)=\frac{1}{2 \pi} \int_{-\infty}^{\infty} \tilde{G}_{X}\left(z, z_{g} ; \omega\right) \tilde{I}_{g}(\omega) e^{j \omega t} d \omega,
$$

where $z_{g}$ is the source position $\left(\vec{r}_{g}=z_{g} \hat{e}_{z}\right), z$ is the observation point and $X(z, t)$ denotes the voltage or current along the line (in the $z$ direction), as a function of the Green's functions employed [ $\tilde{G}_{V}\left(z, z_{g} ; \omega\right)$ or $\tilde{G}_{I}\left(z, z_{g} ; \omega\right)$, related to the voltage or current, respectively]. It should be noted that the space dependence has been absorbed in the Green's function term, while the temporal information is described by Fourier and inverse-Fourier transforms.

Let us consider a simple matched transmission line, as shown in Fig. 5(a) $\left[Z_{g}=Z_{L}=\right.$ $\left.Z_{0}(\omega), \forall \omega\right]$. In this simple case, the transmission line Green's functions for the voltages and 


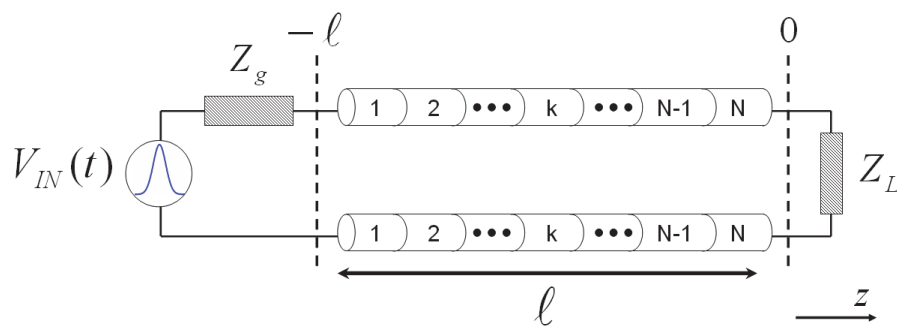

(a)

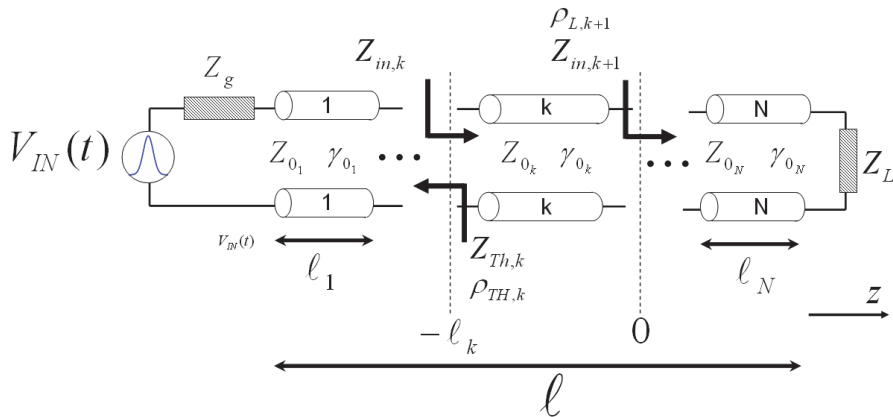

(b)

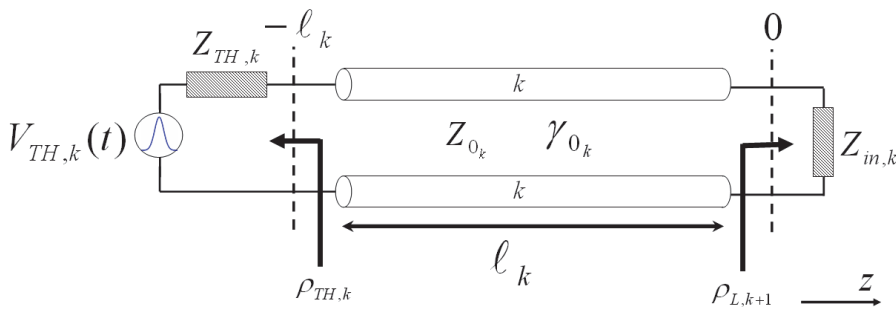

(c)

Fig. 5. Dispersive artificial transmission line excited by a point source generator. (a) Uniform case. The line, composed of $N$ unit cells, is defined by its characteristic impedance $\left[Z_{0}(\omega)\right]$, complex propagation constant $[\gamma(\omega)]$ and length $(\ell)$. (b) Non-uniform case. The line is composed of $N$ uniform transmission line sections. Each $k^{\text {th }}$ section has its own length $\left(\ell_{k}\right)$, characteristic impedance $\left[Z_{0_{k}}(\omega)\right]$ and propagation constant $\left[\gamma_{k}(\omega)\right]$. (c) Thévenin equivalent circuit for the $k^{\text {th }}$ uniform transmission line section. Reprinted with permission from Gómez-Díaz et al. (2010). Copyright 2010, IET.

currents may be expressed as

$$
\begin{aligned}
& \tilde{G}_{V}\left(\vec{r}, \vec{r}_{g} ; \omega\right)=e^{-\gamma(\omega) R}, \\
& \tilde{G}_{I}\left(\vec{r}, \vec{r}_{g} ; \omega\right)=\frac{e^{-\gamma(\omega) R}}{Z_{0}(\omega)},
\end{aligned}
$$


respectively, where $\gamma(\omega)$ is the complex propagation constant (or dispersion relation), $Z_{0}(\omega)$ is the characteristic impedance, and $R=\left|z-z_{g}\right|$ is the distance between the observation point $z$ along the line and the source point $z_{g}$ (generator).

Using these expressions, the voltage or the current along the line can easily be found with Eq. (7). Note that this equation applies to any type of transmission line, including metamaterial CRLH lines, provided that the propagation constant $[\gamma(\omega)]$ is known.

Consider now the more general case of a nonuniform transmission line medium composed of $N$ uniform transmission line sections (or unit-cells), as shown in Fig. 5(b). The sections may be different from each other and may be of different type. Therefore, reflections occur due to the transition between two consecutive cells, and different propagation conditions appear at each cell. The Green's function along the $k^{\text {th }}$ uniform transmission line section $\left(z \in\left[-\ell_{k}, 0\right]\right.$, possibly infinitesimal), including generator and load mismatches, reads

$$
G_{k}\left(z, z^{\prime}=-\ell ; \omega\right)=A(\omega)\left[e^{-\gamma_{k}(\omega) z}+\rho_{l, k}(\omega) e^{\gamma_{k}(\omega) z}\right],
$$

where [see Pozar (2005)]

$$
A(\omega)=\frac{V_{T h, k}(\omega) Z_{i n, k}(\omega)}{Z_{i n, k}(\omega)+Z_{T h, k}} \frac{e^{-\gamma_{k}(\omega) \ell_{k}}}{1-\rho_{l, k}(\omega) \rho_{T h, k}(\omega) e^{-2 \gamma_{k}(\omega) \ell_{k}}},
$$

and

$$
\begin{aligned}
& \rho_{T h, k}(\omega)=\frac{Z_{T h, k}(\omega)-Z_{0_{k}}(\omega)}{Z_{T h, k}(\omega)+Z_{0_{k}}(\omega)}, \\
& \rho_{l, k}(\omega)=\frac{Z_{i n, k+1}(\omega)-Z_{0_{k}}(\omega)}{Z_{i n, k+1}(\omega)+Z_{0_{k}}(\omega)},
\end{aligned}
$$

which was obtained by equating the Green's function evaluated at the input of the $k^{\text {th }}$ section, to the Thévenin's voltage evaluated at the same section $V_{T h, k}$ [see Fig. 5(c)]. By repeatedly applying Eq. (10) from the load to the generator or vice-versa, the voltage as a function of time may be computed at any point along the nonuniform transmission medium.

Finally, note that the use of periodic input signals is important to produce some periodic phenomena or devices, such as the Talbot effect introduced in Azaña \& Muriel (2001) or the UWB resonator presented in Gómez-Díaz et al. (2009a), among many others. For this purpose, the theory previously introduced can easily be extended to consider this type of input signals. In this case, the input signal may be represented in the time domain by

$$
I_{g}^{p}(t)=\sum_{k=-\infty}^{k=+\infty} I_{g}\left(t-k T_{0}\right),
$$

where $T_{0}$ is the period rate. The voltage or current along the transmission line may then be computed by inserting Eq. (14) in Eq. (7). Note that for many input pulses, such as modulated Gaussian pulses, the interchange between the integral and summation operations (related to Eq. (7) and Eq. (14), respectively), allowed by the assumed linearity of the system, will further contribute to reduce the computational cost required by this technique. 


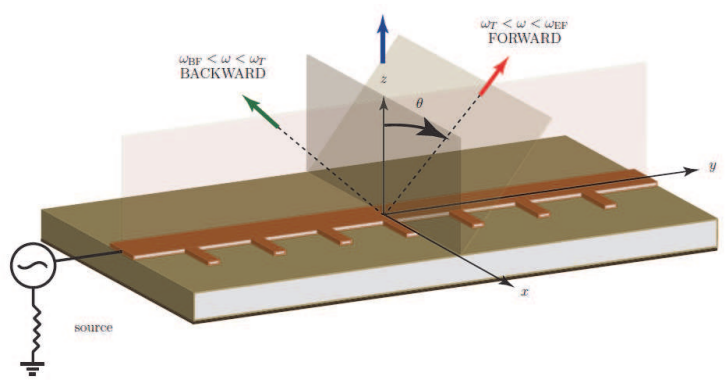

Fig. 6. Illustration of a CRLH LWA. The antenna can be configured to radiate at backwards $\left[\omega<\omega_{T}\right.$ and $\left.\beta(\omega)<0\right]$, forwards $\left[\omega>\omega_{T}\right.$ and $\left.\beta(\omega)>0\right]$ or broadside $\left[\omega=\omega_{T}\right.$ and $\beta(\omega)=0]$.

\subsection{Impulse regime analysis of CRLH leaky-wave antennas}

A CRLH transmission line supports a fast-wave mode [see Oliner \& Jackson (2007)] which penetrates inside the fast-wave region. Therefore a CRLH structure behaves as a leaky-wave antenna when it is excited by a source with a frequency within a range of ( $\left.\omega_{B F}<\omega<\omega_{E F}\right)$, where $\omega_{B F}$ and $\omega_{E F}$ are the fast-wave region limits [see Fig. 4(a) and Caloz \& Itoh (2006)]. Since a CRLH line behaves as a LWA, the direction of the radiated main beam follows the LWA scanning law, which is given by [see Oliner \& Jackson (2007)]

$$
\sin (\theta) \approx \frac{\beta(\omega)}{k_{0}}
$$

In the above equation, $\theta$ is the radiation angle (measured from the perpendicular direction over the CRLH structure), $\beta(\omega)$ is the phase constant, and $k_{0}$ is the free-space wavenumber. Fig. 6 presents an illustration related to the operation principle of a CRLH LWA. As can be seen in the figure, and following Eq. (15), the antenna is able to radiate at backwards [when $\omega<\omega_{T}$ and $\beta(\omega)<0$ ], forwards $\left[\omega>\omega_{T}\right.$ and $\beta(\omega)>0$ ] and broadside $\left[\omega=\omega_{T}\right.$ and $\beta(\omega)=0]$. Therefore, this type of structure is able to provide a full-space scanning, from backfire $\left(\theta=-90^{\circ}\right)$ to endfire $\left(\theta=+90^{\circ}\right)$, including the broadside $\left(\theta=0^{\circ}\right)$ direction. The use of CRLH transmission lines as leaky-wave antennas has led to the development of many radiated-wave applications, most of them in the harmonic regime [see Caloz \& Itoh (2006) and Eleftheriades \& Balmain (2005)].

This subsection proposes an impulse-regime analysis of leaky-wave structures. Even though the proposed study is valid for all types of LWAs, we will focus on CRLH LWA structures because they are broadband in nature and they are able to radiate from backfire to endfire, including the broadside direction [see Caloz \& Itoh (2006)]. As previously pointed out, a CRLH leaky-wave antenna follows the beam-scanning law of Eq. (15). Therefore, each input frequency, which lies inside the fast-wave region, is radiated towards a different direction into space. This situation is explicitly depicted in Fig. 7(a). As can be seen in the figure, there is a unique correspondence between each input frequency $\left[\omega_{x}\right.$, with $\left.\left(\omega_{B F} \leq \omega_{x} \leq \omega_{E F}\right)\right]$ and its associated radiation angle $\left(\theta_{x}\right)$. Therefore, each frequency is mapped into a different angle in space.

According to Eq. (15), if a CRLH LWA is excited by a modulated input pulse, as shown in Fig. 7(b), each spectral component of the signal is radiated towards a different direction 


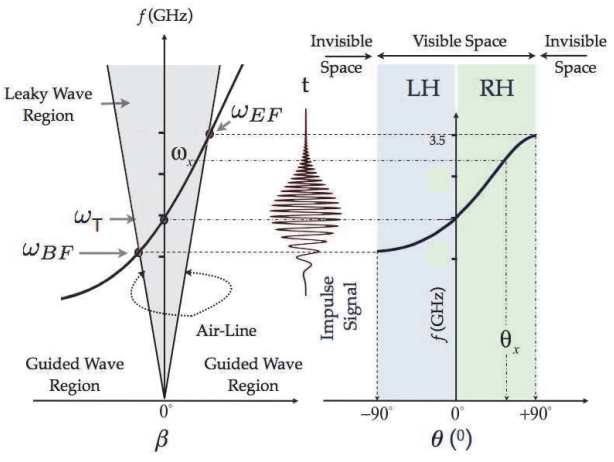

(a)

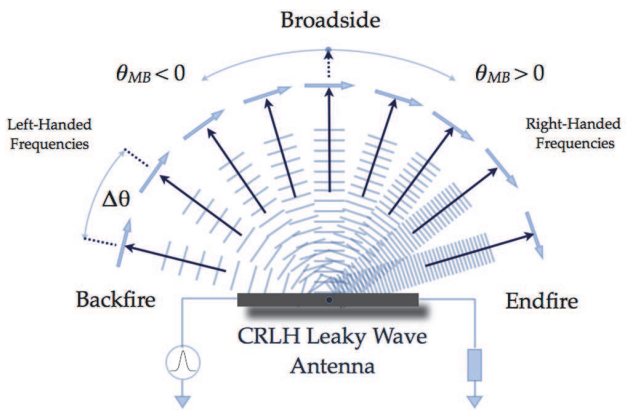

(b)

Fig. 7. Impulse-regime behavior of CRLH LWAs. (a) Frequency-space relationship of a CRLH LWA. The dispersion curve is graphically related to its corresponding beam scanning law. (b) Spectral decomposition of a pulse obtained by the frequency-space mapping property of a CRLH leaky-wave antenna. Reprinted with permission from Gupta, Abielmona \& Caloz (2009). Copyright 2009, IEEE.

in space at any particular instant. Hence, the CRLH LWA performs an instantaneous spectral-to-spatial decomposition of the input pulse. This decomposition allows to discriminate the various spectral components present in the input signal. In this sense, there is clear parallelism between LWAs (which usually operates at microwaves) and diffraction gratings [see Saleh \& Teich (2007)], which usually operate at the optics regime and radiate each spatial input frequency towards a different direction angle in space. The main advantage of CRLH LWA over diffraction gratings is its simple point feeding system as compared to the diffraction gratings, which require plane-wave illumination.

Let us consider a single 1D (or electrically thin) LWA, located along the $z$ axis, as shown in Fig. 8. It is important to clarify the notation employed to describe the situation under analysis. First, the line is defined by a characteristic impedance $\left[Z_{0}(\omega)\right]$, a complex propagation constant $[\gamma(\omega)=\alpha(\omega)+j \beta(\omega)]$, and a total length, $\ell$. Second, the generator which excites the transmission line is placed at the position $\vec{r}_{g}$, with $\vec{r}_{g}=z_{g} \hat{e}_{z}$. Third, any point along the line is denoted as $\vec{r}^{\prime}$, with $\vec{r}^{\prime}=z^{\prime} \hat{e}_{z}$ (note that $z_{\text {start }} \leq z^{\prime} \leq z_{\text {end }}$, see Fig. 8). Besides, note that shall study the far-field radiation of the transmission line towards an observation point $P$ (denoted as $\vec{r}$, with $\vec{r}=x \hat{e}_{x}+y \hat{e}_{y}+z \hat{e}_{z}$ ). Therefore, any observation point P must be located in the far-field region of the transmission line [see Balanis (2005)], fulfilling the far-field radiation condition, which is given by

$$
R_{\text {ant }}=\sqrt{x^{2}+y^{2}+\left(z-\frac{\ell}{2}\right)^{2}}>\frac{2 \ell^{2}}{\lambda_{0}} .
$$

In this last equation, $R_{\text {ant }}$ is the distance between the observation point $P$ (placed at $\vec{r}$ ) and the transmission line, which can approximately be considered as a point source (placed at the center of the line) from a far-field point of view. 


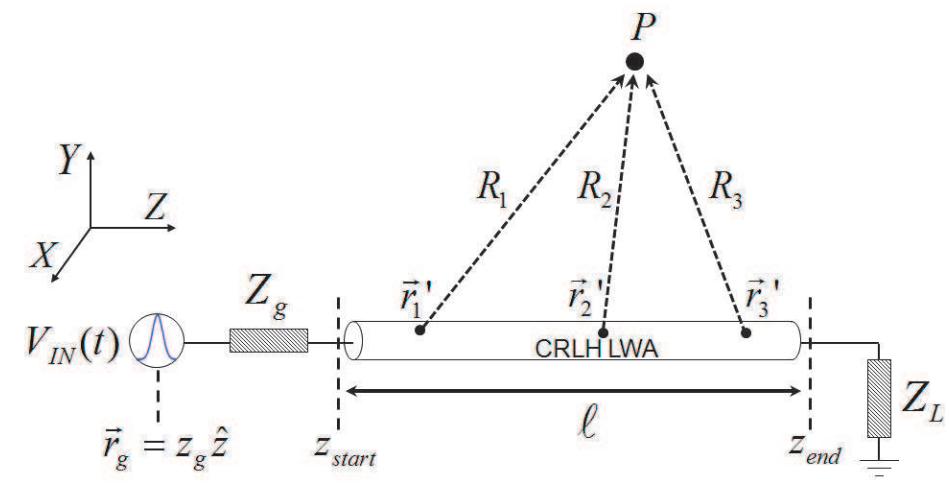

Fig. 8. Sketch of a single 1D CRLH LWA. The electrically thin antenna is considered as a linear wire from a far-field point of view. It is placed along the $z$-axis, it has a length of $\ell=z_{\text {end }}-z_{\text {start }}$, and it is fed by a point generator, placed at $\vec{r}=z_{g} \hat{e}_{z}$.

Let us also assume that the CRLH LWA is excited by a modulated input pulse. In this case, the time-domain theory developed in Section 2.2 for the analysis of impulse-regime CRLH TL can be employed to obtain the current which flows along the structure, which may be expressed as

$$
I\left(z^{\prime}, t\right)=\int_{-\infty}^{\infty} \tilde{G}_{I}\left(z^{\prime}, z_{g}, \omega\right) \tilde{I}_{g}(\omega) e^{j \omega t} d \omega
$$

In this last equation, $\tilde{G}_{I}\left(z^{\prime}, z_{g}, \omega\right)$ represents the transmission line Green's function, related to the current, and $\tilde{I}_{g}(\omega)$ denotes the Fourier transform of the temporal input pulse.

The electric field radiated by the transmission line under study, in the far-field region, is approximately given by

$$
\vec{E}(\vec{r}, \omega) \approx-j \omega \vec{A}(\vec{r}, \omega),
$$

where $\vec{A}$ is the magnetic vector potential. It is important to keep in mind that this potential is related to the physical current which is flowing on the transmission line [see Balanis (2005)]. In the harmonic case, this potential may be expressed as

$$
\vec{A}(\vec{r}, \omega)=\frac{\mu_{0}}{4 \pi} \int_{z_{\text {start }}}^{z_{\text {end }}} I\left(z^{\prime}, \omega\right) \frac{e^{-j k_{0} R}}{R} d z^{\prime} \hat{e}_{z}
$$

where $R$ is the distance between the pair of source-observation points.

However, in the impulse-regime case the CRLH LWA is excited by a temporal pulse. This time-domain behavior of the current induced on the structures propagates into the magnetic vector potential, which now reads

$$
\vec{A}(\vec{r}, t)=\frac{\mu_{0}}{4 \pi} \int_{\omega_{B F}}^{\omega_{E F}} \int_{z_{\text {start }}}^{z_{\text {end }}} I\left(z^{\prime}, \omega\right) \frac{e^{-j k_{0} R}}{R} d \omega d z^{\prime} \hat{e}_{z}
$$


It is important to remark that the frequency integration limits have been modified with respect to Eq. (7). Specifically, the frequency limits directly corresponds to the fast-wave region range $\left(\omega_{B F} \leq \omega \leq \omega_{E F}\right)$. This is because LWAs are only able to radiate spectral components which lies inside this region. Outside of the fast-wave region, this type of structures behaves as a transmission lines as described in Caloz \& Itoh (2006).

Finally, the time-domain electric field radiated by a CRLH LWA, under a far-field assumption, can easily be recovered using

$$
\vec{E}(\vec{r}, t)=\frac{-j \mu_{0}}{4 \pi} \int_{\omega_{B F}}^{\omega_{E F}} \int_{z_{\text {start }}}^{z_{\text {end }}} \omega I\left(z^{\prime}, \omega\right) \frac{e^{-j k_{0} R}}{R} d \omega d z^{\prime} \hat{e}_{z} .
$$

This expression provides the time-domain electric field radiation from a CRLH LWA excited by a modulated input pulse, at any observation point placed in the far-field region. The main features of this closed-form formulation are:

- All CRLH LWA radiation features at far-field are taken into account by using a time-domain current along the structure. This current is closely related to the complex propagation constant of the structure.

- Physical insight into the antenna radiation properties. The Green's function and the current flowing along the structure, related to the CRLH structure, completely define the antenna behavior. These parameters are obtained in closed-form.

- Extremely fast computation, because most expressions are simple and well-behaved integrals (and some of them are analytical for specific input pulses).

- Capability to deal with any type of input pulse, providing a continuous temporal output with unconditional stability.

The proposed approach can characterize complex radiated-wave UWB phenomena and devices. Specifically, this formulation will be employed in the next section to model several systems, such as a real-time spectrum analyzer (RTSA) or a frequency-resolved electrical gating system (FREG). It will be shown that the proposed formulation is able to completely characterize these systems with high efficiency and accuracy .

\section{Optically-inspired phenomena at microwaves}

\subsection{Introduction}

This section explores the impulse-regime phenomenology of CRLH structures and the subsequent theoretical and practical demonstration of several novel optically-inspired phenomena and applications at microwaves, in both, the guided and the radiative regime. The time-domain Green's function approach introduced in Section 2 has opened the door to a very fast, but still accurate, analysis of these novel microwave phenomena and applications, most of them transported from optics, exploiting either the group velocity or the group velocity dispersion parameters of CRLH TL. The study can be divided into two main groups, related to the guided-wave or radiative-wave natures of the proposed phenomena and applications.

The analogy between these phenomena at microwave and their corresponding counterpart at optics [see Saleh E Teich (2007)] is deduced from the dispersive properties of CRLH structures. Specifically, in the guided mode there is a clear parallelism between the dispersive behavior of a CRLH line and an optical component (such as an optical fiber), which is inherently dispersive. Therefore, 
optical phenomena [such as the well-known temporal Talbot effect] can be reproduced at microwaves. In the radiative mode, the beam scanning law of the CRLH LWA is analog to a diffraction grating, where different spectral components are radiated (or diffracted) at different angles causing spatial dispersion. This may be exploited to develop spectrogram analyzers, capable to fully characterize both, in time and frequency, any unknown input signal.

\subsection{Guided-regime}

This subsection presents a study on the phenomenology of pulse propagation along CRLH transmission lines. As previously stated, the CRLH TL represents a general transmission medium, which is highly dispersive, especially in the left-handed frequency range. Next, the temporal Talbot effect [see Azaña \& Muriel (1999)] is introduced, theoretically described and numerically confirmed for the case of CRLH media.

\subsubsection{Pulse propagation along CRLH structures}

The main goal of this subsection is to experimentally validate the theory presented in Section 2. For this purpose, the propagation of a modulated pulse along a CRLH TL is studied. The tunability of the pulse delay as a function of the modulation frequency is demonstrated by measuring the temporal delay from different modulated pulses, leading to a tunable delay system [see Abielmona et al. (2007)]. Then, the dispersive features of the CRLH TL are further demonstrated monitoring the effects of pulse propagation along a matched and mismatched line, in a cell-by-cell fashion.

First, we consider a CRLH transmission line composed of 30 unit cells and with the circuit parameters $C_{R}=1.8 \mathrm{pF}, C_{L}=0.9 \mathrm{pF}, L_{R}=3.8 \mathrm{nH}$ and $L_{L}=1.9 \mathrm{nH}$ (transition frequency $f_{0}=2.55 \mathrm{GHz}$ ), excited by a modulated Gaussian pulse $[\sigma=3.0 \mathrm{~ns}$, following the notation introduced in Saleh \& Teich (2007)]. Fig. 9 shows the time-delayed waveforms obtained by the proposed theory for different carrier frequencies, and by experiments using a real-time oscilloscope (Agilent Infiniium DS0871204B). Excellent agreement is observed between theory and experiments. The group delay response is an important parameter in dispersive systems for analog signal processing applications. This parameter may be computed either in the time domain by determining the time differences between the maxima of the input and output pulses, or in the frequency domain by taking the derivative of the unwrapped phase of the transmission scattering parameter $S_{21}$. Fig. 10 shows the group delay along the same CRLH line and for the same pulse as in Fig. 9, computed by the discussed theory using the first approach, and validated by experiment using both approaches. Again, very good agreement is observed between theory and experiment. The small discrepancies between the two measured results may be explained by the tolerance in the localization of the pulse maxima. As it may be seen from these results and was originally presented in Abielmona et al. (2007), the CRLH transmission line acts as an impulse tunable delay system.

To better visualize the dispersion of a pulse along a CRLH dispersive medium, consider now a CRLH transmission line twice as long as before (60 unit cells), but with the same parameters. To completely validate the discussed theory, this time the line is excited by a modulated square pulse $\left[f_{0}=2.05 \mathrm{GHz}, T=2.2 \mathrm{~ns}\right.$, following the notation of Saleh \& Teich (2007)]. An ABCD matrix approach, described in Caloz \& Itoh (2006), is employed to compute the propagation constant of the line, taking into account the finite number of unit cells in the experiment. The position-time trajectory of the pulse is presented in Fig. 11. Fig. 11(a) shows the computed 


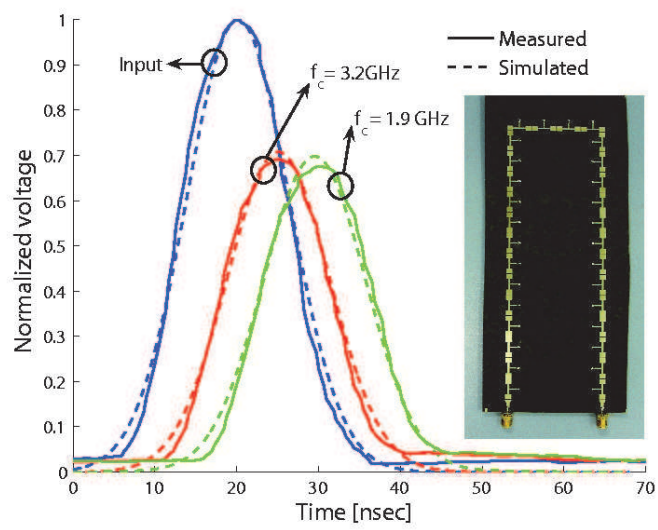

Fig. 9. Time-delayed Gaussian waveforms at the input/output of a CRLH transmission line for different carrier frequencies, obtained with the method proposed in Section 2.2.

Measurement results are also shown for validation. The manufactured CRLH transmission line is shown in the inset. Reprinted with permission from Gómez-Díaz et al. (2009b). Copyright 2009, IEEE.

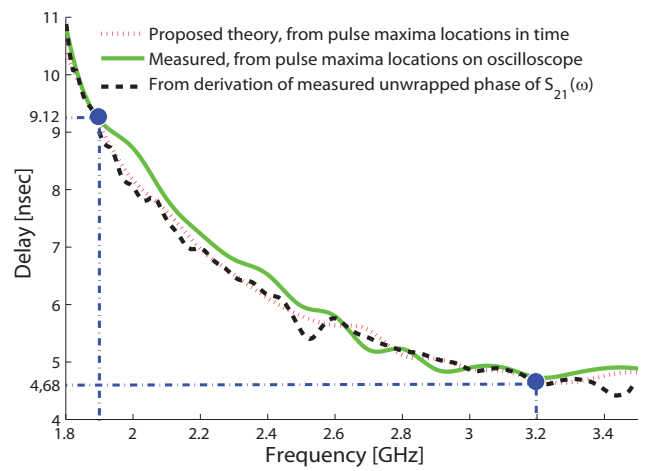

Fig. 10. Time delay versus modulation frequency, using the time difference between the maxima of the input and output pulses along the same CRLH line as in Fig. 9. Measured data using both, the same procedure as before and unwrapping the phase of $S_{21}(\omega)$, are also shown for validation. The delays obtained in Fig. 9 for $f_{c}=3.2 \mathrm{GHz}$ and $f_{c}=1.9 \mathrm{GHz}$, which are 4.68 and 9.12 ns, correspond to the two highlighted points. Reprinted with permission from Gómez-Díaz et al. (2009b). Copyright 2009, IEEE.

results, from which two observations may be made: i) at the end of the line, the temporal width of the pulse has increased by a factor of 5 (at $50 \%$ of the magnitude), ii) the edges of the square envelope have been rounded off by the band-pass filtering response of the CRLH line. The small ripples near the end of the structure are explained by the Gibbs effect on the input pulse due to the finite computational interval and resolution. Fig. 11(b) shows the measured result using a high-impedance probe connected to the oscilloscope. The abrupt decrease of the voltage magnitude after the $30^{\text {th }}$ cell is due to the fact that the 60 -cell experimental line is 


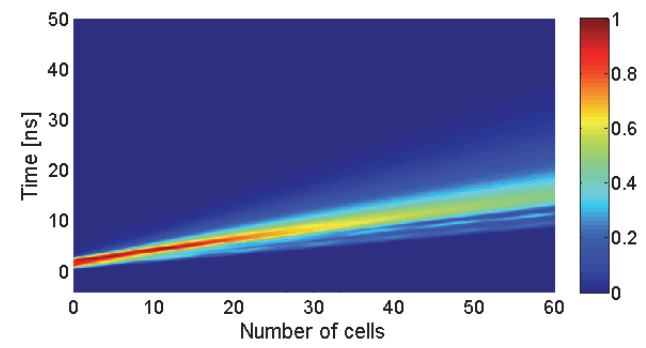

(a)

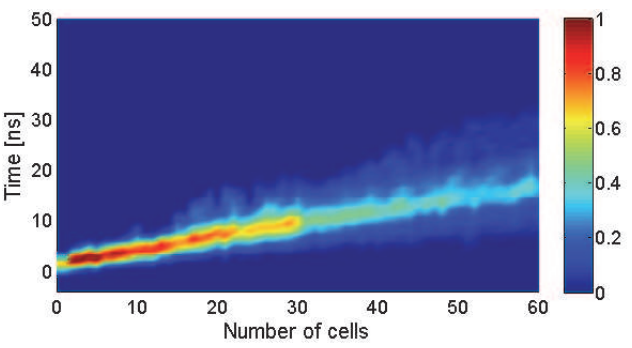

(b)

Fig. 11. Propagation of a modulated square pulse $\left(f_{0}=2.05 \mathrm{GHz}, T \stackrel{(b)}{=} 2.2 \mathrm{~ns}\right)$ along a matched CRLH transmission line. The line includes 60 unit cells of length $p=2.0 \mathrm{~cm}$ and the circuital parameters are $C_{R}=1.8 \mathrm{pF}, C_{L}=0.9 \mathrm{pf}, L_{R}=3.8 \mathrm{nH}$ and $L_{L}=1.9 \mathrm{nH}$. (a) Simulation. (b) Measurement. Reprinted with permission from Gómez-Díaz et al. (2009b). Copyright 2009, IEEE.

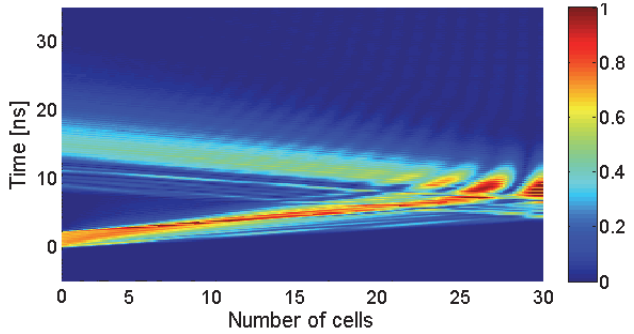

Fig. 12. Propagation of a modulated square pulse $\left(f_{0}=2.05 \mathrm{GHz}, T \stackrel{(\mathrm{b})}{=} 2.2 \mathrm{~ns}\right)$ along an

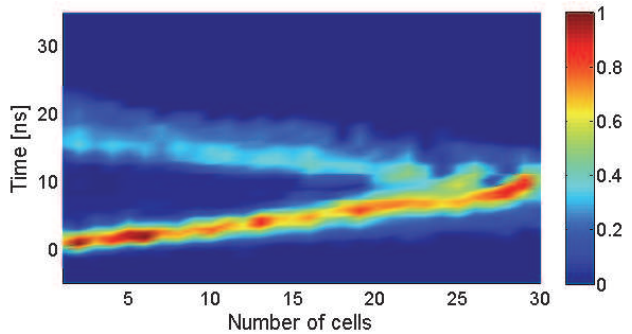

Fig. 12. Propagation of a modulated square pulse $\left(f_{0}=2.05 \mathrm{GHz}, T \stackrel{(\mathrm{b})}{=} 2.2 \mathrm{~ns}\right)$ along an open-ended CRLH transmission line. The line is identical to that of Fig. 11 except that it includes only 30 unit cells. (a) Simulation. (b) Measurement. Reprinted with permission from Gómez-Díaz et al. (2009b). Copyright 2009, IEEE.

in fact constituted of two cascaded 30-cell lines with a small loss in the interconnection. The agreement with theory is reasonable, considering the tolerances of the measurement setup.

Finally, let us investigate the effects of the reflection of a pulse at a discontinuity of a CRLH transmission line. For this purpose, the line is open-ended and it is excited by the same modulated square pulse as in the previous case. The propagation and the reflection of the pulse as a function of space and time is presented in Fig. 12. Again, good agreement is observed between the theory [Fig. 12(a)] and the experiment [Fig. 12(b)]. It is worth noticing that an interesting interference pattern, between propagating and reflected pulses, occurs near the discontinuity.

\subsubsection{Temporal Talbot effect}

The Talbot effect is a periodic constructive interference pattern produced by a dispersive transmission medium with second-order dispersion for a periodic input signal. It was first reported by H. F. Talbot in 1836 for the case of a source with periodic spatial variation [see Talbot (1836)]. The temporal counterpart of this effect [see Azaña \& Muriel (1999) or Azaña \& Muriel (2001)] occurs when a time-periodic signal is propagating along the same kind of medium. An input pulse train with temporal period $T_{0}$ and pulse width $\Delta T$ is 
replicated at the position $n z_{T}(n \in \mathbb{N})$, where $z_{T}$ is called the Talbot distance (or self-imaging distance). Also, an increased repetition rate of $m$ pulses per $T_{0}$ is obtained at the fractional distances $z_{f}=(s / m) z_{T}$ (where $s, m \in \mathbb{N}$ ), provided that $(s / m)$ is an irreducible fraction, and under the condition that: $m<T_{0} / \Delta T$ [see Azaña \& Muriel (2001)]. The temporal Talbot effect has been employed mainly in the optical regime, for applications such as the generation of signals with ultrahigh repetition rate $(\mathrm{THz})$ from slower ranges $(\mathrm{GHz})$, as shown in Azaña \& Muriel (2001), or pulse compression, as presented in detail in Berger et al. (2004).

At microwaves, this phenomenon is more difficult to reproduce because it is difficult to obtain a second-order dispersive medium at this frequency region. However, the recent introduction of CRLH TLs [see Caloz \& Itoh (2006)], which provides a dispersive broadband behavior, may lead to novel scenarios where the temporal Talbot effect and their subsequent applications can be reproduced. This section provides a theoretical demonstration of the temporal Talbot effect in CRLH media, including a full-wave validation of the phenomena at microwaves. Furthermore, the conditions for the phenomena existence in CRLH media are stated, in connection with potential device applications.

Intuitively, the temporal Talbot effect occurs when a periodic pulse signal is transmitted through a second order dispersion medium where the neighboring pulses interfere as a result of dispersion so as to produce new temporal components. For the analysis, let us consider a single modulated pulse, denoted by $\Psi(t)=\Psi_{0}(t) e^{j \omega_{0} t}$, where $\Psi_{0}(t)$ is a slowly varying envelope. The periodic signal is then represented in the time domain, at the position $z=0$, as

$$
A(z=0, t)=\sum_{n=-\infty}^{n=+\infty} \Psi\left(t-n T_{0}\right),
$$

where $T_{0}$ is the repetition rate of the signal. In the spectral domain, the periodic signal becomes discrete, and it may be expressed as

$$
\tilde{A}(z=0, \omega)=\omega_{r} \sum_{n=-\infty}^{n=+\infty} \tilde{\Psi}\left(\omega=n \omega_{r}\right) \delta\left(\omega-n \omega_{r}\right),
$$

where $\omega_{r}=2 \pi / T_{0}$ is the spectral repetition frequency.

On the other hand, the transfer function of a lossless CRLH TL is given by

$$
\tilde{H}(z, \omega)=e^{-j \beta(\omega) z},
$$

where $\beta(\omega)$ is the CRLH TL propagation constant. This propagation constant, around the CRLH TL transition frequency, may be approximated as

$$
\beta(\omega)=\frac{\omega}{\omega_{R}^{\prime}}-\frac{\omega_{L}^{\prime}}{\omega}
$$

where $\omega_{R}^{\prime}=p / \sqrt{L_{R} C_{R}}$ and $\omega_{L}^{\prime}=1 / \sqrt{p^{2} L_{L} C_{L}}$, as described in Caloz \& Itoh (2006). In the right handed side of Eq. (25), the first term provides a simple time delay (or linear frequency phase), whereas the second order term is responsible for the line dispersion. Eq. (25) can be further expanded, employing Taylor series, around a modulation frequency $\left(\omega_{0}\right)$ as

$$
\beta(\omega)=\beta_{0}+\beta_{1}\left(\omega-\omega_{0}\right)+\frac{1}{2} \beta_{2}\left(\omega-\omega_{0}\right)^{2}+O\left(\omega^{3}\right),
$$


where the term $O\left(\omega^{3}\right)$ is related to the order of the error committed in the approximation, and the term $\beta_{n}$ is defined as

$$
\beta_{n}=\left.\left[\frac{\beta^{n}(\omega)}{\partial \omega^{n}}\right]\right|_{\omega=\omega_{0}} .
$$

Note that Eq. (26) is only valid for the case of narrowband pulses (centered at the frequency $\omega_{0}$ and with bandwidth $\Delta \omega$ ).

Employing Eq. (26), the transfer function of the CRLH TL $\left[\tilde{H}\left(\omega^{\prime}\right)=\tilde{H}\left(\omega=\omega_{0}+\omega^{\prime}\right)\right.$, where a change of variable has been introduced from $\omega$ to $\omega^{\prime}$ ] takes the form

$$
\tilde{H}\left(z, \omega^{\prime}\right)=\exp \left\{-j\left[\beta_{0}+\beta_{1} \omega^{\prime}+\frac{1}{2} \beta_{2} \omega^{\prime 2}\right] z\right\} .
$$

In order to derive the Talbot distance, only the third expansion term of the exponential is considered. This is because the first two terms do not provide any information related to the Talbot distance [which is only due to second order dispersion, see Azaña \& Muriel (2001)]. Specifically, the first term is related to the modulation frequency of the pulse and does not carry any information about the envelope, and the second term represent the group delay (or retarded frame) of the signal.

Using Eq. (23) and Eq. (28), the spectrum of the signal at the distance $z$ can be written as

$$
\begin{aligned}
\tilde{A}\left(z, \omega^{\prime}\right)= & \omega_{r} \sum_{n=-\infty}^{n=+\infty} \exp \left\{-j \frac{\beta_{2} \omega^{\prime 2} z}{2}\right\} \tilde{\Psi}\left(n \omega_{r}\right) \delta\left(\omega^{\prime}-n \omega_{r}\right)= \\
& \omega_{r} \sum_{n=-\infty}^{n=+\infty} \exp \left\{-j \frac{\beta_{2} z}{2}\left(\frac{2 \pi n}{T_{0}}\right)^{2}\right\} \tilde{\Psi}\left(n \omega_{r}\right) \delta\left(\omega^{\prime}-n \omega_{r}\right) .
\end{aligned}
$$

Note that the appearance of $\omega^{\prime}$ squared term in this equation is due to the quadratic phase factor in the spectral response of the CRLH TL dispersive medium.

Eq. (29) reveals that the Talbot effect [i.e., $\left.\tilde{A}_{z}(z, \omega)=\tilde{A}(z=0, \omega)\right]$ occurs under the condition

$$
\frac{\beta_{2} z}{2}\left(\frac{2 \pi n}{T_{0}}\right)^{2}=\pi p,
$$

where $p \in \mathbb{N}$. The case of the first integer Talbot distance $(p=1)$ is given by

$$
z_{T}=\frac{T_{0}^{2}}{2 \pi\left|\beta_{2}\right|}=\frac{T_{0}^{2} \omega_{0}^{3}}{4 \pi \omega_{L}^{\prime 2}},
$$

where the identity $\beta_{2}=-2 \omega_{L}^{\prime 2} / \omega_{0}^{3}$ [see Eq. (27)] has been employed.

As demonstrated in Azaña \& Muriel (2001), the Talbot distance can also be obtained at fractionary distances given by $z_{f}=(s / m) z T$, where $s$ and $m$ are irreducible integers. At this fractionary distance, the periodic input signal is also self-imaged but with an increase repetition rate by a factor of $m$. This phenomenon corresponds to the fractionary Talbot effect [see Azaña \& Muriel (2001)]. It is important to note that in the case of a CRLH TL, the Talbot distance can be tuned externally by modifying the parameters $T_{0}$ and $\omega_{0}$, without changing the intrinsic parameters of the line. 


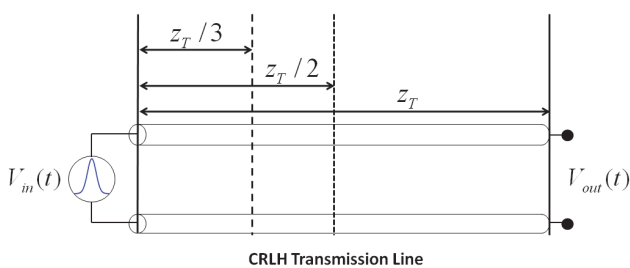

(a)

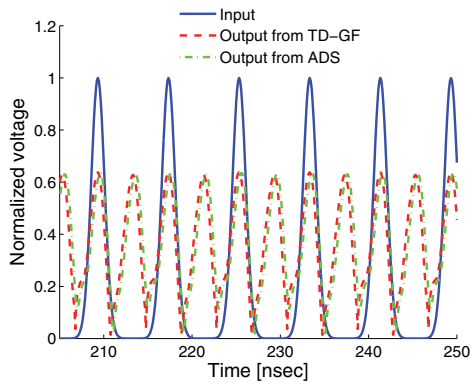

(c)

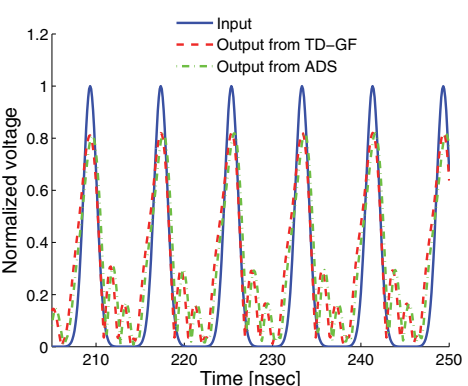

(b)

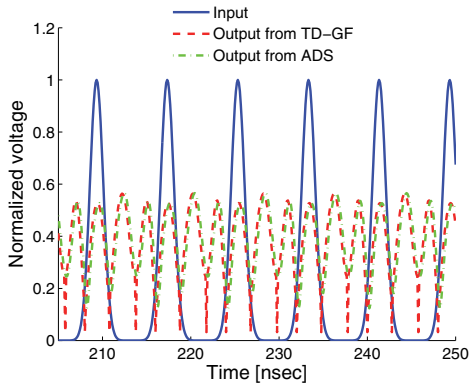

(d)

Fig. 13. Talbot repetition rate multiplication effect. a) CRLH TL with length corresponding to the basic Talbot distance $z_{T}$. b) Reconstruction of the original pulse train at the Talbot distance $z_{T}$. c) Repetition rate doubling at the distance $z_{T} / 2$. d) Repetition rate tripling at the distance $z_{T} / 3$. Results obtained from the proposed approach, and validated using the commercial software ADS@. Reprinted with permission from Gómez-Díaz et al. (2009b). Copyright 2009, IEEE.

After theoretically deriving the Talbot distance related to CRLH TLs, the phenomena will be verified using the numerical technique proposed in Section 2.2 and commercial full-wave simulations. Consider a balanced lossless CRLH transmission line with circuital parameters $C_{R}=C_{L}=1.0 \mathrm{pF}$ and $L_{R}=L_{L}=2.5 \mathrm{nH}$. A modulated train of Gaussian pulses, with temporal width of $\sigma=0.75 \mathrm{~ns}$ and period rate of $T_{0}=8 \mathrm{~ns}$ is used to excite the line shown in Fig. 13(a). Fig. 13(b) presents the input pulse train and the output pulse train at the Talbot distance $z_{T}$. The reconstruction of the initial train of pulses is confirmed, although a small disagreement, due to higher order terms (greater than 2) of the CRLH dispersion relation, is observed between two consecutive pulses. Fig. 13(c) and Fig. 13(d) show the input and output voltages at the fractional Talbot distances of $z_{T} / 2$ and $z_{T} / 3$, respectively. The effect of pulse multiplication is thus clearly confirmed, while the distortion is smaller because less higher-order dispersion effects occur over a shorter distance of propagation.

The practical implementation of Talbot devices based on CRLH transmission lines depends on the technology employed. For instance, with the CRLH parameters used in Fig. 13, a microstrip implementation with a typical unit cell size of $1 \mathrm{~cm}$ would lead to a Talbot distance $z_{T}$ of around 17 meters. This is unpractical, specially due to the losses. However, using 
multilayer technology [see Horii et al. (2005)], this distance may be dramatically reduced to sizes in the order of several centimeters, while even lower sizes apply for pulse rate repetition multiplication. This decrease in length also decreases the total amount of losses, allowing the Talbot effect to be applied in practical situations, such as the generation of signals with ultrahigh repetition rate or pulse compression [see Berger et al. (2004)].

\subsection{Radiative-regime}

The full-space scanning capabilities of CRLH LWAs are exploited in this Section to achieve the spectral-spatial decomposition of an input broadband signal. This property is then applied to the development of a real-time spectrum analyzer [RTSA, see Gupta, Abielmona \& Caloz (2009)] and a frequency resolved electrical gating system [FREG, see Gupta, Gómez-Díaz \& Caloz (2009)], which are UWB applications able to fully characterize, in both time and frequency, an unknown signal. These systems are fully modeled by the technique presented in Section 2.3, which constitutes an ideal tool to provide not only a fast numerical system characterization, but a deep physical insight into the electromagnetic properties of the devices.

\subsubsection{Real time spectrogram analyzer (RTSA)}

A real time spectrogram analyzer (RTSA) is a device able to provide a joint time-frequency representation of an unknown input signal. This device is specially useful in modern UWB systems, as described in Ghavami et al. (2007), where ultra-fast transient signals are involved. The common output of a RTSA system is an spectrogram [see Cohen (1989)], which is a 2-D plot of a signal where the energy distribution is related to an image in a time-frequency plane. The joint time-frequency representation provides information related to the temporal evolution of each spectral component, and the exact amplitude and location in time of each frequency. It is important to mention that spectrograms suffer from the fundamental "uncertainty principle" limitation, which states

$$
\Delta t \Delta f \geq \frac{1}{2}
$$

where $\Delta f$ is the bandwidth of the gated signal and $\Delta t$ is the gate duration. This implies an inherent trade off between time and frequency resolution for all spectrograms.

There are two main approaches to obtain the spectrogram of an unknown input test signal at microwave frequencies. The first and more usual technique is based on the use of digital components, which performs the short-time Fourier transform (STFT) [see Oppenheim (1996)] of the unknown input signal. However, the use of this approach at microwaves has the important drawback of requiring fast processors and large memories, which limits its use to input signals of just a few hundreds megahertz and temporal resolutions of only a few microseconds. The second option, purely analog, is based on the use of a bank of filters, as described in Amin \& Feng (1995). The main disadvantage of this approach is that it requires a very large number of channels and extremely narrow-band filters, which is specially difficult to achieve at high frequencies. Consequently, this option is very complex and expensive.

In this context, a novel RTSA based on the spectral-spatial decomposition of CRLH LWAs was proposed in Gupta, Abielmona \& Caloz (2009). In this approach, the CRLH LWA provides an analog implementation of the STFT, in a similar way as it has been implemented in the optical regime. A schematic of the CRLH LWA RTSA system is shown in Fig. 14. The description of the system behavior is as follows. First, the spectral-spatial decomposition of the CRLH LWA 


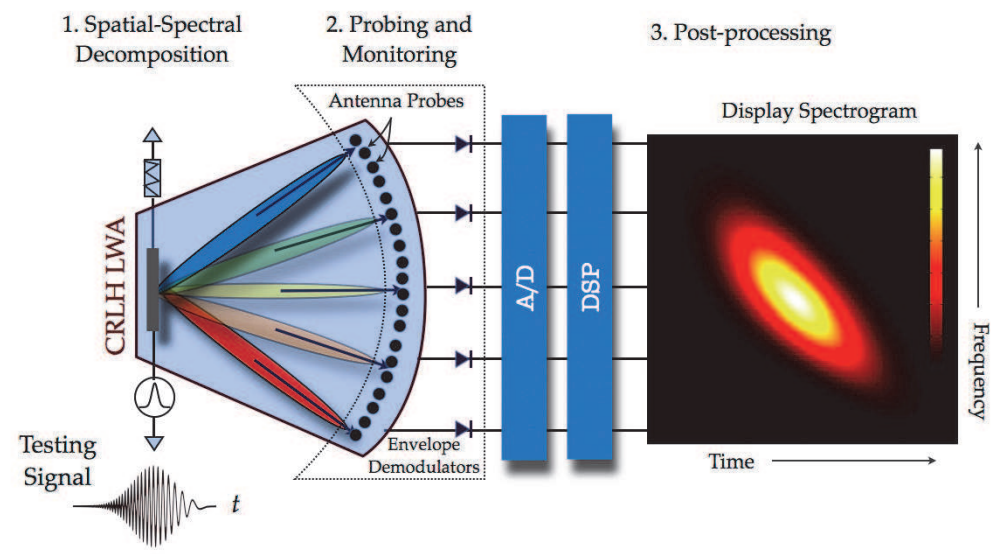

Fig. 14. Analog real-time spectrogram analyzer showing the CRLH LWA, the antenna probes, the envelope detectors, the A/D converters, the DSP block, and the display with the spectrogram. Reprinted with permission from Gupta, Abielmona \& Caloz (2009). Copyright 2009, IEEE.

is employed to discriminate the frequency components of the input test signal (see Section 2.3). Second, a set of probes (antenna receivers) monitor the time variation of each frequency component. Finally, a postprocessing step performs the analog/digital (A/D) conversion, the data processing and the display of the spectrogram.

The analog CRLH LWA RTSA system provides several advantages and benefits as compared with other RTSAs at microwave frequencies. First, this approach is completely analog and real time. Therefore, there is not requirement of large memories and fast processors, just a light postprocessing stage. Second, the same RTSA system can use different CRLH LWAs, in order to be flexible and to cover several frequency ranges. The use of new technologies to fabricate CRLH LWAs allows the use of a wide variety of input signals, from microwave up to potentially millimeter-wave frequencies. Third, the CRLH LWA RTSA system is inherently broadband, and it can be designed to process the $100 \%$ of an input signal bandwidth.

On the other hand, the CRLH LWA RTSA system has also to deal with some drawbacks. First, it requires a far-field probe configuration, which makes the system relatively large. However, the system can possibly be compacted employing near-field to far-field transformations. Second, the physical length of the LWA represents a space-gating mechanism which controls both, the temporal and the frequency resolution of the resulting spectrograms. This is clearly shown in Fig. 15, where a slice of the signal's spatial waveform is presented on and radiating from the antenna. And third, the time and frequency resolution also depends on the number of detectors, and their associated response time and sampling frequency. Therefore, there is a fundamental tradeoff between time and frequency resolutions.

In order to characterize this system, full-wave commercial software may be employed. However, the generation of the output results are extremely time consuming due to the complexity of the system and to the temporal nature of the analysis. An interesting and efficient alternative is to employ the theory developed for the modeling of impulse-regime radiation (see Section 2.3), to fully characterize the CRLH LWA RTSA system. For the sake of 


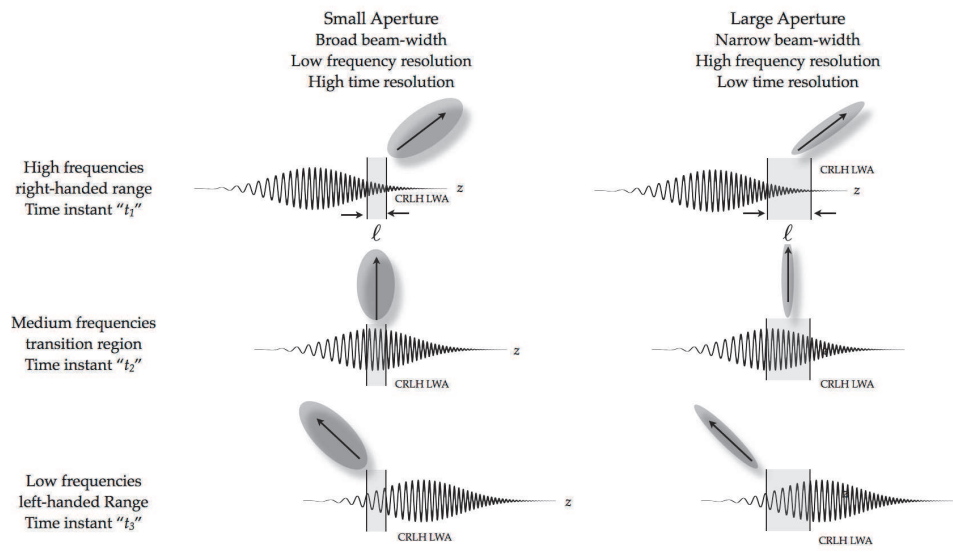

Fig. 15. Impact of the LWA size $\ell$ on the time-frequency resolution of the spectrograms generated by the an analog CRLH LWA RTSA.

validation, let us consider a complete RTSA system based on a CRLH LWA which is composed of 32 unit cells of length $p=1.0 \mathrm{~cm}$, with circuital parameters of $C_{R}=C_{L}=1.0 \mathrm{pF}$ and $L_{R}=L_{L}=2.5 \mathrm{nH}$. In order to complete the analog RTSA system, a total of number of 181 observation probes are placed in a semi-circular configuration, following the configuration of Fig. 14. The first step to model the RTSA is to perform the calibration of the system. This is necessary to compensate for the different power levels received at each probe [as described in Gómez-Díaz et al. (2010)], due to the directivity variation with frequency of this type of antennas. For the calibration, a narrow-band signal is modulated to the different fast-wave frequencies [following Eq. (15) (scanning law)] and subsequently radiated by the LWA. Then, the maximum power received at each probe is stored, obtaining a normalization rule for this particular system configuration. In our example, the calibration data is shown in Fig. 16(a). After the RTSA system has been calibrated, it can be efficiently used to obtain spectrograms of an unknown input signal. For the method validation, the actual temporal and frequency information of an input test signal, previously known, is employed.

Let us consider now that the CRLH LWA is fed by a signal composed of three modulated Gaussian pulses. The first pulse has a positive-chirp modulation (which means that the modulation frequency is increasing with time), and the third pulse has a negative-chirp modulation. The spectrogram obtained with the proposed method, after calibration, is depicted in Fig. 16(b), including an additional graph showing the analytical temporal representation of the signal. As can be observed in the figure, the spectrogram follows the signal variations in time (the three pulses are clearly observable) and also, simultaneously, in frequency. It is especially interesting to observe the transition between two consecutive pulses, where frequencies corresponding to different pulses appear at the same instant.

The method proposed here is able to perform a quick (about 30 seconds) and accurate modeling of the RTSA system, with deep insight into the CRLH LWA time-radiation properties, and avoiding the extremely time-consuming analysis required in full-wave simulations (usually between $8-12$ hours). Therefore, it provides a fast tool to configure an RTSA system and to determine a priory the range of input signals which can accurately be characterized for a given CRLH LWA. 


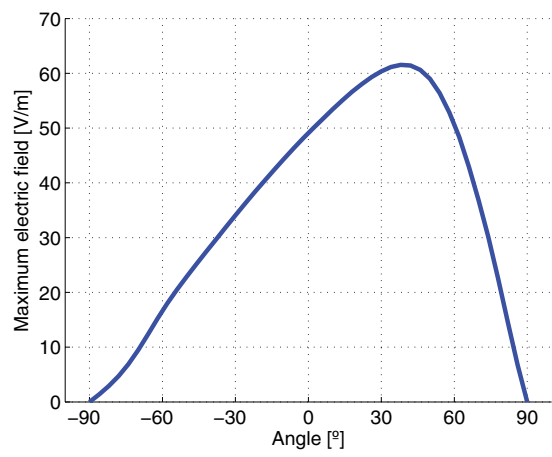

(a)

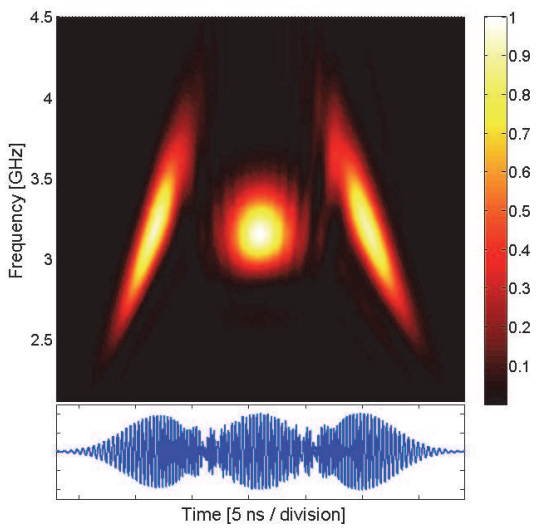

(b)

Fig. 16. Results obtained from an RTSA system based on a CRLH LWA composed of 32 unit cells of length $p=1.0 \mathrm{~cm}$, with circuital parameters of $C_{R}=C_{L}=1.0 \mathrm{pF}$ and $L_{R}=L_{L}=2.5 \mathrm{nH}$. (a) Maximum electric field obtained at the different positions of the probes, used for the calibration of the RTSA system. (b) Normalized spectrogram of a three chirp-modulated Gaussian pulses signal, with chirp parameters $C=-[10,0,10]$, modulation frequency $f_{0}=3.19 \mathrm{GHz}$ and temporal width $\sigma=1.0 \mathrm{~ns}$, computed with the proposed technique. The inset shows the analytical time response of the signal. Reprinted with permission from Gómez-Díaz et al. (2010). Copyright 2010, IET.

Finally, experimental results from a RTSA prototype are included for a complete system validation and to confirm the accuracy of the proposed modeling technique. For this purpose, a CRLH LWA fabricated in microstrip technology and composed of $14-0.8 \mathrm{~cm}$ long unit cells, with circuital parameters $C_{R}=1.29 \mathrm{pF}, C_{L}=0.602 \mathrm{pF}, L_{R}=3.0 \mathrm{nH}$ and $L_{L}=1.4 \mathrm{nH}$, is employed. A photo of the fabricated antenna is depicted in Fig. 17(a), whereas a comparison of the measured and simulated scattering parameters is shown in Fig. 17(b). Furthermore, Fig. 17(c) presents a simulation-measurements comparison of the antenna dispersion relationship. As can be observed, the CRLH LWA presents its transition frequency at $3.745 \mathrm{GHz}$. The antenna fast-wave frequency region starts at about $3.1 \mathrm{GHz}$ (related to backfire radiation), and it is extended until $4.7 \mathrm{GHz}$ (related to endfire radiation). Note that several resonances occur within the fast-wave frequency region (close to endfire), degrading the antenna performance. These resonances are due to internal resonances of the interdigital capacitors employed in the antenna prototype, and they are not considered in the circuital model of the LWA.

In order to measure the spectrogram of the test signals, a single receiver was used and rotated around the circular far-field trajectory of the system (specifically, between $\theta=-80^{\circ}$ and $\theta=$ $80^{\circ}$ with increments of $5^{\circ}$ ). The RTSA system is calibrated employing a linear frequency ramp. After calibration, the CRLH LWA is excited by a modulated Gaussian pulse, with FWHM of 3.5 ns. Fig. 18 presents a comparison between simulations (obtained by the proposed time-domain Green's function approach) and measurements, as a function of the pulse modulation frequency. It can be observed that a very good agreement is achieved in all cases. First, the pulse modulation frequency is set to $3.3 \mathrm{GHz}$, which corresponds to backwards radiation. This is clearly shown in the spectrograms of Fig. 18(a) and Fig. 18(b). Then the 


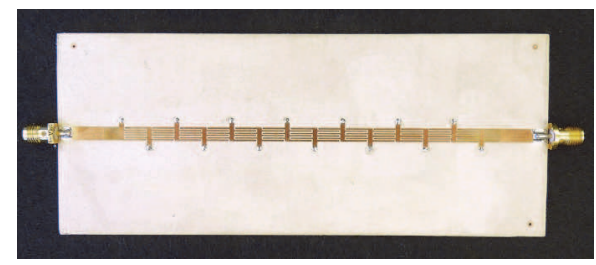

(a)

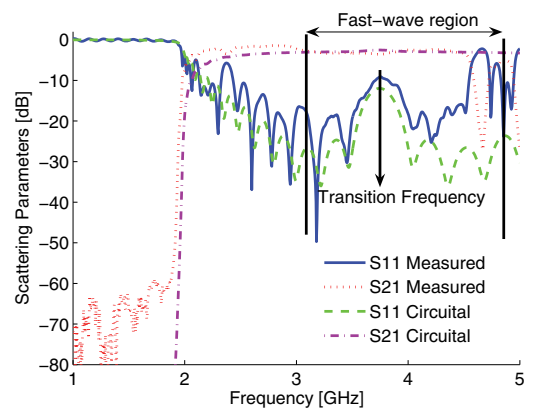

(b)

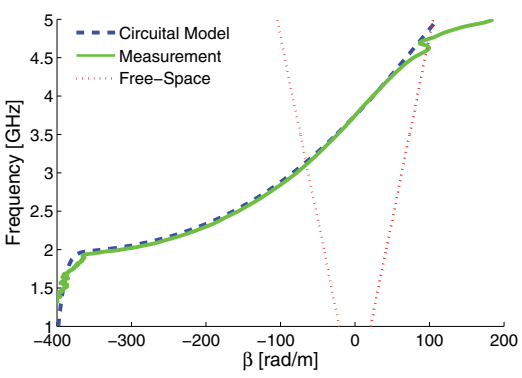

(c)

Fig. 17. 1D CRLH LW antenna composed by $14-0.8 \mathrm{~cm}$ - long cells, with circuital parameters $C_{R}=1.29 \mathrm{pF}, C_{L}=0.602 \mathrm{pF}, L_{R}=3.0 \mathrm{nH}$ and $L_{L}=1.4 \mathrm{nH}$. a) Photo of a microstrip CRLH LWA prototype. b) Scattering parameters. c) Dispersion relation. Reprinted with permission from Gómez-Díaz et al. (2009c). Copyright 2009, American Institute of Physics.

modulation frequency is set to the CRLH LWA transition frequency, which corresponds to broadside radiation. As expected, the obtained spectrograms [see Fig. 18(c) and Fig. 18(d)] confirm the change in frequency. Finally, pulse modulation frequency is set to $4.2 \mathrm{GHz}$, which corresponds to a forward direction. Again the spectrograms show the changes in the pulse frequency, experimentally verifying the RTSA system and confirming the usefulness of the proposed theoretical approach to model this type of analog systems.

\subsubsection{Frequency resolved electrical gating system (FREG)}

Analog CRLH LWA RTSAs generate the spectrogram of an unknown input signal in real-time, using the spectral-spatial decomposition property of the leaky-wave antenna, with minimal requirements on computational resources. This system provides important benefits over any digital RTSAs at microwave frequencies. However, as explained in the previous section, the time and frequency resolution of the generated spectrograms directly depends on the physical length of the CRLH LWA, which is fixed in a given system. Therefore, a particular antenna can only handle a limited range of input signals, which must fulfil specific time and frequency constrains. This imposes an important limitation to the CRLH LWA RTSA systems.

This section proposes a novel analog approach to obtain spectrograms, where the hardware dependence is suppressed, at the cost of the requirement of periodicity of the input signals. This approach is inspired from a similar system known in optics as frequency resolved optical gating (FROG) [see Trebino (2002)], where a self-gating principle is applied to provide close to ideal spectrograms for arbitrary test signals. Here, we propose a microwave counterpart of 


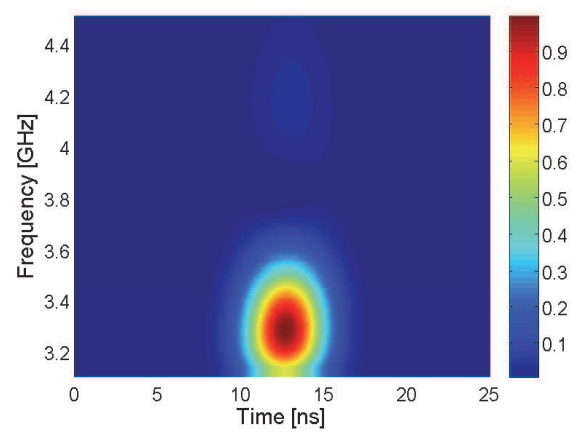

(a)

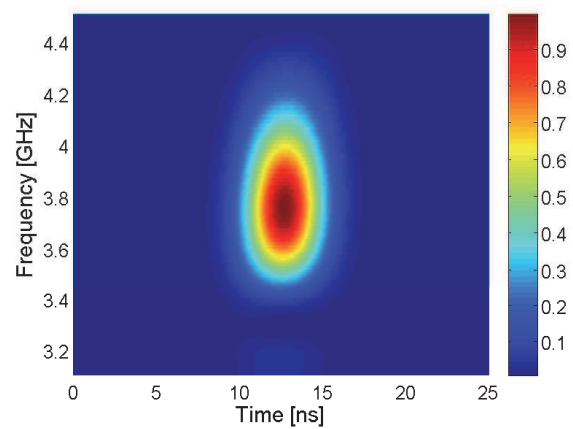

(c)

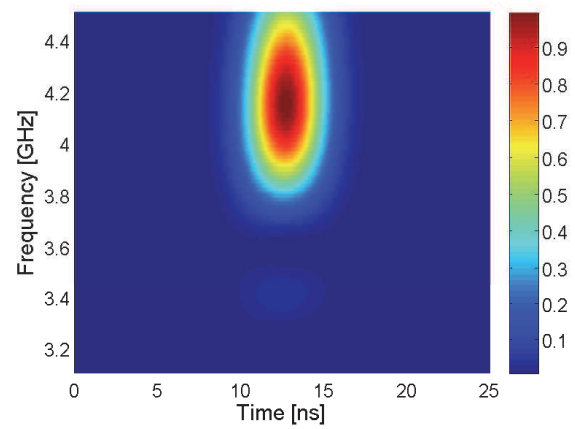

(e)

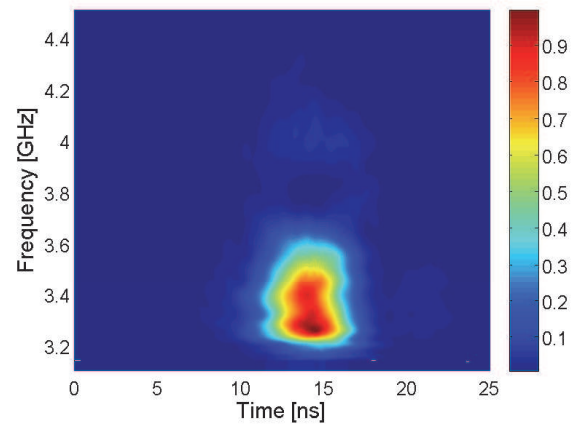

(b)

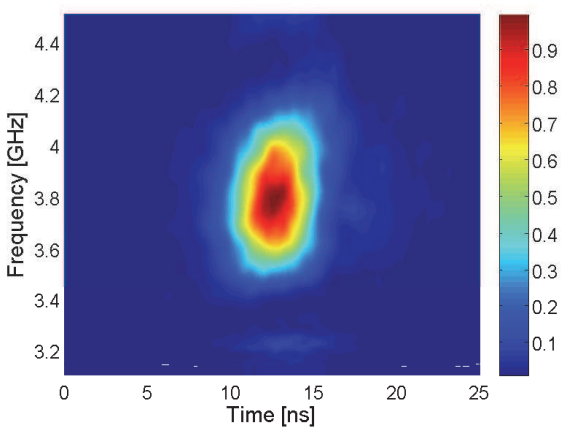

(d)

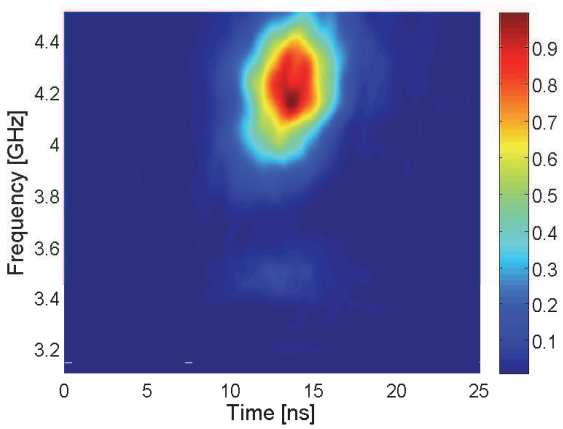

(f)

Fig. 18. Spectrograms obtained by the proposed RTSA model (figures on the left) and by experiments (figures on the right), employing the CRLH LWA of Fig. 17. A modulated Gaussian pulse with $F W H M=3.5$ ns feeds the antenna. The pulse modulation frequency is set to 3.3, 3.745 and $4.2 \mathrm{GHz}$, corresponding to backward [(a) and (b)], broadside [(c) and (d)] and forward [(e) and (f)] radiation, respectively. 


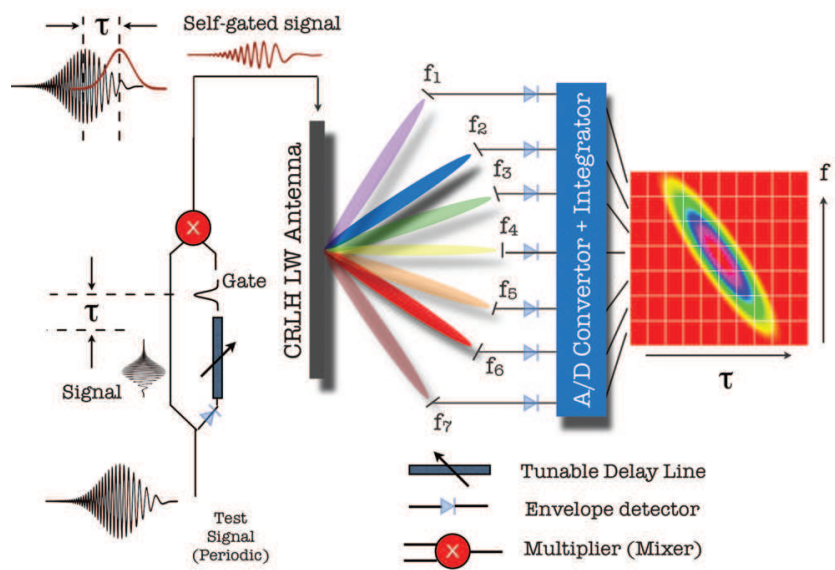

Fig. 19. Proposed frequency resolved electrical gating (FREG) system. Reprinted with permission from Gupta, Gómez-Díaz \& Caloz (2009). Copyright 2009, EuMC.

the FROG system, which is termed frequency resolved electrical gating (FREG). This system is very useful for the measurement and characterization of fast-varying non-stationary UWB signals and ultrashort pulses.

In order to compute a spectrogram of a signal $x(t)$, a temporal gating function $g(t)$ is required [see Cohen (1989)]. Using a self-gating approach, instead of using a separate time signal as the gate function, the envelope of the testing signal itself is used as the gating function, i.e. $g(t)=$ $|x(t)|$. The spectrogram of a signal $x(t)$ is then obtained as

$$
S(\tau, \omega)=\left|\int_{-\infty}^{\infty} x(t)\right| x(t-\tau)\left|e^{-j \omega t} d t\right|^{2} .
$$

The proposed FREG system, based on this self-gating principle and on the spectral-spatial decomposition property of the CRLH LWA, is depicted in Fig. 19. The testing signal, whose spectrogram is to be generated, is split into two channels. One of the channels is envelope detected and passed through a tunable delay line. The two channels are then mixed together. The mixer thus performs the self-gating process at a given time delay instant $\tau$. This self-gated signal is then injected into a CRLH LWA which spectrally resolves it in space. Once the frequency components are separated in space, antennas circularly placed in the far-field of the LWA receive the different frequency components corresponding their angular position. All the received signals are then digitized and summed, before being stored for spectrogram display. This process is repeated for different values of the time delay $\tau$ so that the entire test signal is scanned, according to Eq. (33), until the spectrogram is fully constructed. Since, the beam scanning law of CRLH LWA is nonlinear in nature, a final post-processing step is required to linearize the spectrogram.

The proposed system exhibits significant advantages over the analog RTSA system and purely digital systems. Due to the self-gating process, neither the time nor the frequency resolutions of the generated spectrogram depend on the physical length of the antenna. The time and frequency resolutions are thus dependent only on the time signal itself and the hardware 


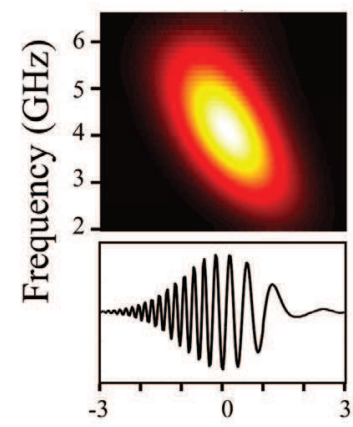

(a)

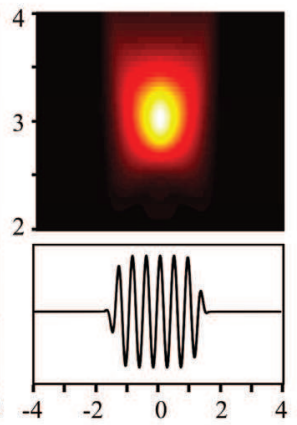

(b)

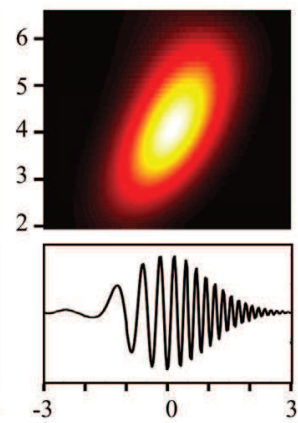

(c)

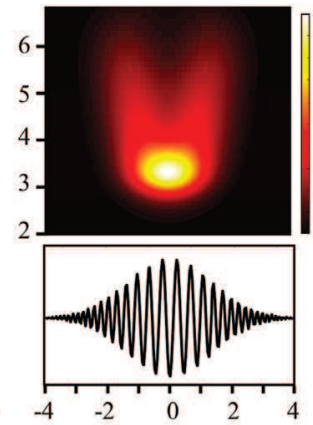

(d)

Fig. 20. Simulated spectrograms. a) Down-chirped gaussian pulse $\left(C_{1}=-10, C_{2}=0\right.$, $\left.f_{0}=4 \mathrm{GHz}\right)$. b) Non-chirped super-gaussian pulse $\left(C_{1}=C_{2}=0, f_{0}=3 \mathrm{GHz}\right)$. c) Up-chirped gaussian pulse $\left(C_{1}=+10, C_{2}=0, f_{0}=4 \mathrm{GHz}\right)$. d) Cubically chirped gaussian pulse $\left(C_{1}=0, C_{2}=0.25 \times 10^{28}\right)$. All pulse have a FWHM duration of 1 ns with a initial pulse offset of $t_{0}=6.5 \mathrm{~ns}$, and are described using Eq. (34). Reprinted with permission from Gupta, Gómez-Díaz \& Caloz (2009). Copyright 2009, EuMC.

dependence spectrogram is suppressed. The LWA simply plays a role of spectral decomposer which, when longer (higher directivity), provides better separation of frequencies in space.

The choice of the gate duration is an important parameter to achieve an optimal time-frequency resolution in the spectrogram. An optimal gate duration for pulses with dominating phase variations is given by $T_{g} \approx 1 / \sqrt{2\left|\phi^{\prime \prime}(t)\right|}$, where $\phi^{\prime \prime}(t)$ is the second time derivative of phase [see Cohen (1989)]. This duration permits the resolution of the fastest phase variations. For general pulse measurement, a gate duration as short as the testing signal itself or slightly shorter is thus desirable. Since the FREG system is based on self-gating, the gate duration is close to optimal and the corresponding spectrograms are ideal, as demonstrated in Trebino (2002).

Moreover, the proposed system being analog in nature, neither require fast processors nor huge memory buffers, which avoid placing a heavy computational burden on the system. Furthermore, the system is frequency scalable and sufficiently broadband to handle a wide variety of UWB signals. As mentioned above, the length of the LWA controls the spectral decomposition of the gated signal, which is improved as the physical length of the antenna is increased. Finally, since it uses a multi-shot measurement procedure, where the testing signal is gated several times with different time delays $\tau$, the proposed FREG system requires a periodic input signal. This is the main limitation of the FREG system.

In order to simulate the proposed FREG system, the time-domain Green's functions approach presented in Section 2.3 is employed. The role of this theory is to model the transient CRLH LWA behavior, which provides the spectral-spatial decomposition property and is a key component of the proposed FREG system. Then, the other components of the system are implemented as follows. The envelope of the testing signals are numerically obtained and used as a gating function. The tunable time delay between the replica of the test signal and the gate signal is applied, and the mixer, which performs the self-gating operation, is modeled 
by a simple mathematical product. The resulting signal is then fed into the CRLH LWA. As a final stage, the temporal radiation computed at the probe locations are integrated as a function of the gate delay $(\tau)$, in order to recompose the desired spectrogram.

Once the numerical model of the system is complete, spectrograms obtained from various test signals are computed. For this purpose, consider a 16-cell CRLH LWA with the circuital parameters $C_{L}=C_{R}=1 \mathrm{pF}, L_{L}=L_{R}=2.5 \mathrm{nH}$ and a unit cell size of $p=2 \mathrm{~cm}$, easily implemented in metal-insulator-metal (MIM) technology [see Abielmona et al. (2007)]. The various modulated testing pulses are gaussian and super gaussian-type signals which follows the equation

$$
v(t)=C_{0} \operatorname{Re}\left\{\exp \left[j 2 \pi f_{0} t-\frac{1}{2}\left(1+j C_{1}\right)\left(\frac{t-t_{0}}{\sigma}\right)^{2 m}+j C_{2}\left(t-t_{0}\right)^{3}\right]\right\},
$$

where $C_{0}$ controls the pulse amplitude, $t_{0}$ is the time offset, $\sigma$ is related to the pulse duration, $C_{1}$ and $C_{2}$ control the linear and quadric chirp modulation, $f_{0}$ is the pulse modulation frequency and $m$ is an integer.

Fig. 20 shows FREG-generated spectrograms. Figs. 20(a) and (c) show the spectrograms of a down-chirped and up-chirped gaussian pulses, respectively. A faithful representation of a linear instantaneous frequency variation is obtained. The spectrogram of a modulated un-chirped super-gaussian pulse is shown in Fig 20(b), where the occurrence of all the frequency components of the signal at the same time instant are clearly seen. Finally, Fig. 20(d) shows the spectrogram of a cubically chirped (down and up) gaussian pulse. The high frequency components occurring at two different times, characteristic of cubically chirped pulses, can be clearly identified. These few examples demonstrate the capability of the proposed FREG system to analyze a wide variety of non-stationary signals.

It is important to point out that a full-wave simulation of the FREG system is extremely time-consuming. Specifically, the FREG system requires multiple analysis of the impulse-regime response of a CRLH LWA, fed by different input signals. Since each of these analysis lasts between $8-10$ hours, the simulation of a complete FREG spectrogram may easily lasts few days, which is completely prohibitive. On the other hand, the use of the time-domain Green's functions approach reduces this time to a few $(5-8)$ minutes. Furthermore, the use of this numerical tool provides a deep insight into the physics of the system, including an electromagnetic modeling of the antenna and a clear understanding of each step of the proposed FREG system.

Finally, a comparison between the FREG and the RTSA systems is given in Fig. 21. The goal of this analysis is to point out the advantages and disadvantages of each method. For the comparison, the same input pulse feeds the RTSA and FREG systems, which are based on identical CRLH LWAs. In the comparison, the number of unit cells $N$ (with size $p=1.56 \mathrm{~cm}$ and circuital parameters of $C_{L}=C_{R}=1 \mathrm{pF}$ and $L_{L}=L_{R}=2.5 \mathrm{nH}$ ) of the antenna is modified to perform several tests. The spectrogram results are then given as a function $N$, i.e. as a function of the total length of the antenna $(\ell=N \cdot p)$. For the test, a modulated Gaussian pulse is employed (with $f_{0}=3.0 \mathrm{GHz}$ and $\sigma=0.5 \mathrm{~ns}$ ). In the figure, the results from the FREG system are placed on the left, whereas the spectrograms computed by the RTSA system are located on the right. First, we set in both systems an antenna with $N=5$ unit cells, obtaining the spectrograms shown in Fig. 21(a) and Fig. 21(b). This antenna is physically very short, which turns out into a very low directivity. This leads to a very bad frequency resolution 


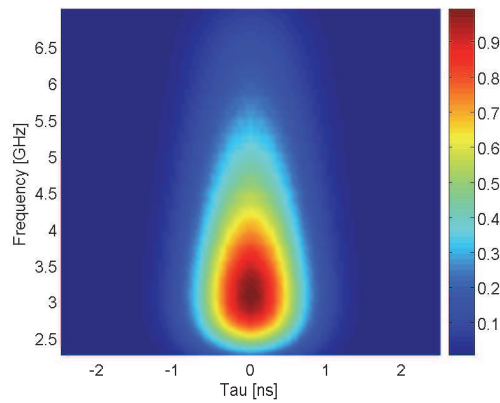

(a)

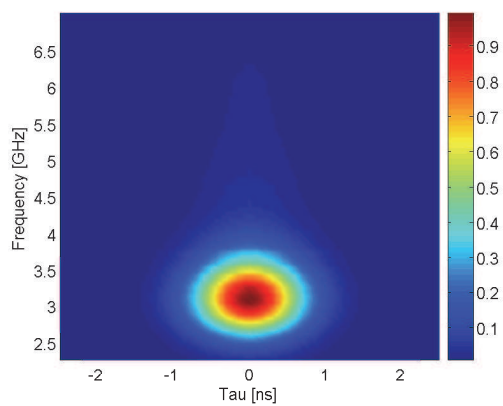

(c)

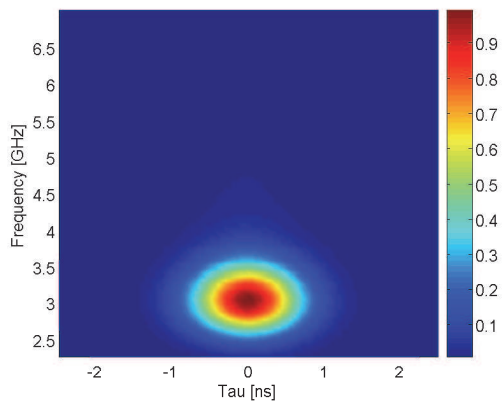

(e)

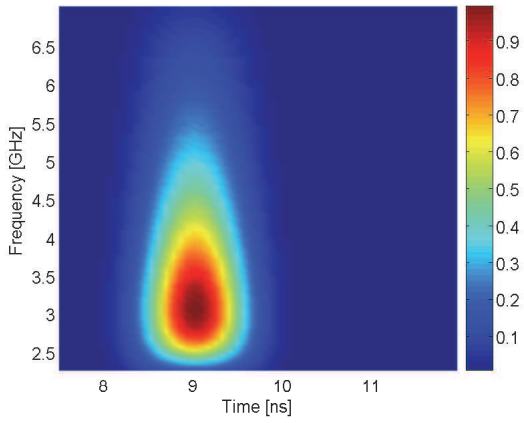

(b)

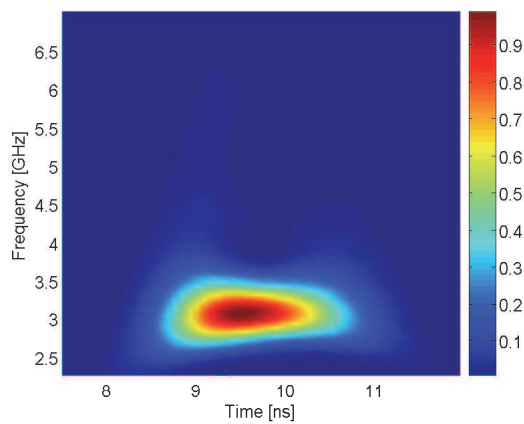

(d)

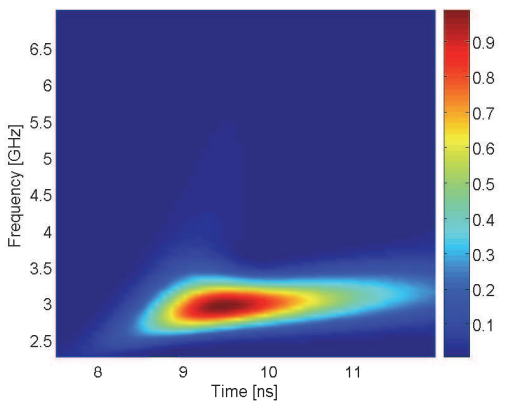

(f)

Fig. 21. Spectrograms obtained by the proposed FREG (figures on the left) and RTSA (figures on the right) systems, based on identical CRLH LWAs for the different tests. The antennas are composed of different numbers of $N$ cells, with length $p=1.56 \mathrm{~cm}$ and circuital parameters of $C_{L}=C_{R}=1 \mathrm{pF}$ and $L_{L}=L_{R}=2.5 \mathrm{nH}$. A modulated Gaussian pulse feeds the systems $\left(f_{0}=3.0 \mathrm{GHz}, \sigma=0.5 \mathrm{~ns}\right)$. The resulting spectrograms are given for the case of $N=5[(\mathrm{a})$ and (b)], $N=20[(\mathrm{c})$ and (d) $]$ and $N=40[(\mathrm{e})$ and (f)] unit cells. 
in both spectrograms. On the other hand, this antenna provides an excellent time-gating trade-off, because the energy is instantaneously radiated, almost without propagation along the structure, leading to a large time resolution. Thereby, the use of a very short antenna leads to generally erroneous spectrograms, due to the wide detection of frequencies which are not part of the input pulse. Second, we modify the CRLH LWA antenna, including now a total of $N=20$ unit cells. This configuration provides a good frequency resolution in both systems, while the temporal resolution is deteriorated in the RTSA system (due to the use of a longer antenna). The resulting spectrograms are depicted on Fig. 21(c) (FREG) and Fig. 21(d) (RTSA). As it can be observed, the FREG system provides a completely realistic spectrogram, which faithfully reproduces the input signal in terms of frequency and time (location and spreading). On the other hand, the spectrogram obtained by the RTSA has a good frequency resolution, but has some problems dealing with the temporal duration of the pulse. As previously commented, this problem is due to the propagation of the input pulse as it is being radiated, as graphically illustrated in Fig. 15. And third, we simulate the FREG and RTSA systems based on the same CRLH LWA, but composed now of $N=40$ unit cells. The results are shown in Fig. 21(e) (FREG) and Fig. 21(f) (RTSA). The spectrogram obtained using the FREG system is quite similar to the previous FREG spectrogram ( $N=20$ unit cells), keeping the temporal characteristics but improving the frequency resolution (because a longer antenna provides higher directivity). All relevant features of the input modulated Gaussian pulse, in terms of frequency and time, can easily be extracted from this spectrogram. However, the RTSA system provides a completely wrong result. This is because of the excessive length of the CRLH LWA, which completely destroy the temporal resolution of the system.

The above comparison demonstrates that the proposed FREG system presents important advantages over the RTSA system, specially in terms on temporal resolution, being able to characterize any unknown UWB input signal. Furthermore, this comparison has shown that the RTSA system can only deal with signals whose frequency and temporal characteristics are -at least overall- previously known. On the other hand, the main constrains of the FREG system are the complex equipment required, the requirement of a periodic input signal, and the fact that it is not a completely real-time system.

\section{Conclusions}

This chapter has introduced an impulse-regime analysis of metamaterial-type transmission lines and antennas. Specifically, a novel formulation, based on Fourier transformations, has been proposed to describe pulse propagation along dispersive linear CRLH lines. The proposed theory is capable to model complex impulse-regime phenomena, such as dispersion, in a simple, accurate and fast way. Then, the method has been extended to consider CRLH leaky-wave antennas, allowing a fast and accurate analysis of the far-field radiation of these structures in time-domain. The proposed formulation has then been applied to the development of novel phenomena and applications in the microwave domain, most of them transported from optics. Instead of the usual magnitude engineering and filter design, a dispersion or phase engineering has been applied. In this approach, the dispersive nature and subsequent impulse-regime properties of CRLH structures have been exploited to obtain novel phenomena/applications. Each phenomenon or application proposed has theoretically been described, numerically verified, and in most of the cases, experimentally demonstrated. The shift from narrow band systems (mostly used in the past) to ultra wide band systems, required by current high date rate wireless communication systems, suggests that the 
forthcoming decades will experience a major interest on this dispersive engineering approach, providing new, novel and more exciting effects and devices at microwaves.

\section{References}

Abielmona, S., Gupta, S. \& Caloz, C. (2007). Experimental demonstration and characterization of a tunable CRLH delay line system for impulse/continuous wave, IEEE Microwave and Wireless Components Letters 17(12): 864-866.

Abielmona, S., Gupta, S., Nguyen, H. V. \& Caloz, C. (2008). Dispersion engineered impulse regime memataterial devices, Proc. XXIXth Assembly of Union Radio Science International (URSI), Chicago, IL, USA.

Amin, G. \& Feng, K. D. (1995). Short-time fourier transforms using cascade filter structures, IEEE Transaction on Circuits and Systems II, Analog Digital Signal Processing 20(3): 631-641-408.

Azaña, J. \& Muriel, M. A. (1999). Technique for multiplying the repetition rates of periodic trains of pulses by means of a temporal self-imaging effect in chirped fiber gratings, Optics Lett. 24: 1672-1674.

Azaña, J. \& Muriel, M. A. (2001). Temporal self-imaging effects: theory and application for multiplying pulse repetition rates, IEEE J. Sel. Top. Quantum Electron 7: 728-744.

Balanis, C. A. (2005). Antenna Theory: Analysis and Design. 3rd Edition., John Wiley and Sons.

Barton, G. (1989). Elements of Green's Functions and Propagation, Oxford Science Publications.

Berger, N. K., Levit, B. S., Bekker, A. \& Fischer, B. (2004). Compression of periodic optical pulses using temporal fractional talbot effect, IEEE Photonics Technology Letters 16(8): 1855-1857.

Caloz, C. (2009). Perspectives on EM metamaterials, Materials Today 12(3).

Caloz, C. \& Itoh, T. (2006). Electromagnetic Metamaterials: Transmission Line Theory and Microwave Applications., Wiley and IEEE Press.

Cohen, L. (1989). Time-frequency distributions-a review, Proceedings of the IEEE 77(2): 941-981.

Collin, R. E. (1991). Field Theory of Guided Waves, IEEE Press, Piscataway, N.J.

Duran-Sindreu, M., Velez, A., Aznar, F., Bonache, J. \& Martin, F. (2009). Application of open split ring resonators and open complementary split ring resonators to the synthesis of artificial transmission lines and microwave passive components, IEEE Transactions on Microwave Theory and Techniques 57(2): 3395-3403.

Eleftheriades, G. (2009). EM transmission-line metamaterials, Materials Today 12(3).

Eleftheriades, G. V. \& Balmain, K. G. (eds) (2005). Negative-Refraction Metamaterials: Fundamental Principles and Applications, Wiley \& IEEE Press, Hoboken, NJ.

Felsen, L. B. (1969). Transients in dispersive media, part I: Theory, IEEE Transactions on Antennas and Propagation 17: 191-200.

Ghavami, M., Michael, L. B. \& Kohno, R. (2007). UWB Signals and Systems in Communication Engineering, J. Wiley \& Sons.

Gómez-Díaz, J. S., Gupta, S., Álvarez-Melcón, A. \& Caloz, C. (2009a). Impulse-regime CRLH resonator for tunable pulse rate multiplication, Radio Science 44(doi:10.1029/2008RS003991): 1-9.

Gómez-Díaz, J. S., Gupta, S., Álvarez-Melcón, A. \& Caloz, C. (2009b). Investigation on the phenomenology of impulse-regime metamaterial transmission lines, IEEE Transactions on Antennas and Propagation 57(12): 4010-4014. 
Gómez-Díaz, J. S., Gupta, S., Álvarez-Melcón, A. \& Caloz, C. (2009c). Tunable talbot imaging distance using an array of beam-steered metamaterial leaky-wave antennas, Journal of Applied Physics 106: 084908-9.

Gómez-Díaz, J. S., Gupta, S., Álvarez-Melcón, A. \& Caloz, C. (2010). Effcient time-domain análisis of highly-dispersive linear and non-linear metamaterial waveguide and antenna structures, IET Microwaves, Antennas and Propagation 4(10): 1617-1625.

Gupta, S., Abielmona, S. \& Caloz, C. (2009). Microwave analog real-time spectrum analyzer (rtsa) based on the spatial-spectral decomposition property of leaky-wave structures, IEEE Transactions on Microwave Theory and Techniques 57(12): 4010-4014.

Gupta, S., Gómez-Díaz, J. S. \& Caloz, C. (2009). Frequency resolved electrical gating principle for UWB signal characterization using leaky-wave structures, 39th European Microwave Conference, Rome, Italy.

Horii, Y., Caloz, C. \& Itoh, T. (2005). Super-compact multilayered left-handed transmission line and diplexer application, IEEE Transactions on Microwave Theory and Techniques 53(4).

Marques, R., Martín, F. \& Sorolla, M. (eds) (2008). Metamaterials with Negative Parameters: Theory, Design and Microwave Applications, Wiley, Hoboken, NJ.

Oliner, A. A. \& Jackson, D. R. (2007). Leaky-wave antennas, in J. L. Volakis (ed.), Antenna Engineering Handbook, 4 edn, McGraw-Hill, New York.

Oppenheim, A. V. (1996). Signals and Systems, Prentice Hall.

Paul, C. R. (2007). Analysis of Multiconductor Transmission Lines, $2^{\text {nd }}$ edition edn, Wiley-IEEE Press.

Pipes, L. A. \& Harvill, L. R. (1971). Applied Mathematics for Engineers and Physicist, $3^{\text {rd }}$ edn, McGraw Hill.

Pozar, D. (2005). Microwave Engineering, $3^{\text {rd }}$ edn, John Wiley and Sons.

Saleh, B. E. A. \& Teich, M. C. (2007). Fundamentals of Photonics, 2nd edition edn, Wiley-Interscience.

Talbot, H. F. (1836). Facts relating to optical science no. IV, Philos. Mag. 9: 401-407.

Trebino, R. (ed.) (2002). Frequency-Resolved Optical Gating: The Measurement of Ultrashort Laser Pulses, Springer. 


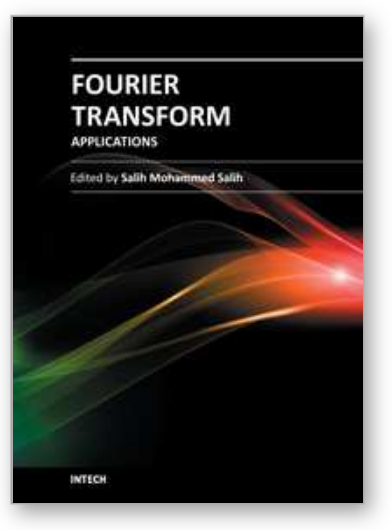

\author{
Fourier Transform Applications \\ Edited by Dr Salih Salih
}

ISBN 978-953-51-0518-3

Hard cover, 300 pages

Publisher InTech

Published online 25, April, 2012

Published in print edition April, 2012

The book focuses on Fourier transform applications in electromagnetic field and microwave, medical applications, error control coding, methods for option pricing, and Helbert transform application. It is hoped that this book will provide the background, reference and incentive to encourage further research and results in these fields as well as provide tools for practical applications. It provides an applications-oriented analysis written primarily for electrical engineers, control engineers, signal processing engineers, medical researchers, and the academic researchers. In addition the graduate students will also find it useful as a reference for their research activities.

\title{
How to reference
}

In order to correctly reference this scholarly work, feel free to copy and paste the following:

J. Sebastian Gomez-Diaz, Alejandro Alvarez-Melcon, Shulabh Gupta and Christophe Caloz (2012). ImpulseRegime Analysis of Novel Optically-Inspired Phenomena at Microwaves, Fourier Transform Applications, Dr Salih Salih (Ed.), ISBN: 978-953-51-0518-3, InTech, Available from: http://www.intechopen.com/books/fouriertransform-applications/impulse-regime-analysis-of-novel-optically-inspired-phenomena-at-microwaves

\section{INTECH}

open science | open minds

\section{InTech Europe}

University Campus STeP Ri

Slavka Krautzeka 83/A

51000 Rijeka, Croatia

Phone: +385 (51) 770447

Fax: +385 (51) 686166

www.intechopen.com

\section{InTech China}

Unit 405, Office Block, Hotel Equatorial Shanghai

No.65, Yan An Road (West), Shanghai, 200040, China

中国上海市延安西路65号上海国际贵都大饭店办公楼 405 单元

Phone: +86-21-62489820

Fax: +86-21-62489821 
(C) 2012 The Author(s). Licensee IntechOpen. This is an open access article distributed under the terms of the Creative Commons Attribution 3.0 License, which permits unrestricted use, distribution, and reproduction in any medium, provided the original work is properly cited. 